\title{
CONJUGATE AND CUT TIME IN THE SUB-RIEMANNIAN PROBLEM ON THE GROUP OF MOTIONS OF A PLANE*
}

\author{
YURI L. SACHKOV ${ }^{1}$
}

\begin{abstract}
The left-invariant sub-Riemannian problem on the group of motions (rototranslations) of a plane SE(2) is studied. Local and global optimality of extremal trajectories is characterized. Lower and upper bounds on the first conjugate time are proved. The cut time is shown to be equal to the first Maxwell time corresponding to the group of discrete symmetries of the exponential mapping. Optimal synthesis on an open dense subset of the state space is described.
\end{abstract}

Mathematics Subject Classification. 49J15, 93B29, 93C10, 53C17, 22E30.

Received March 23, 2009.

Published online August 11, 2009.

\section{INTRODUCTION}

This work is devoted to the study of the left-invariant sub-Riemannian problem on the group of motions of a plane. This problem can be stated as follows: given two unit vectors $v_{0}=\left(\cos \theta_{0}, \sin \theta_{0}\right), v_{1}=\left(\cos \theta_{1}, \sin \theta_{1}\right)$ attached respectively at two given points $\left(x_{0}, y_{0}\right),\left(x_{1}, y_{1}\right)$ in the plane, one should find an optimal motion in the plane that transfers the vector $v_{0}$ to the vector $v_{1}$, see Figure 1 . The vector can move forward or backward and rotate simultaneously. The required motion should be optimal in the sense of minimal length in the space $(x, y, \theta)$, where $\theta$ is the slope of the moving vector.

The corresponding optimal control problem reads as follows:

$$
\begin{aligned}
& \dot{x}=u_{1} \cos \theta, \quad \dot{y}=u_{1} \sin \theta, \quad \dot{\theta}=u_{2}, \\
& q=(x, y, \theta) \in M \cong \mathbb{R}_{x, y}^{2} \times S_{\theta}^{1}, \quad u=\left(u_{1}, u_{2}\right) \in \mathbb{R}^{2}, \\
& q(0)=q_{0}=(0,0,0), \quad q\left(t_{1}\right)=q_{1}=\left(x_{1}, y_{1}, \theta_{1}\right), \\
& l=\int_{0}^{t_{1}} \sqrt{u_{1}^{2}+u_{2}^{2}} \mathrm{~d} t \rightarrow \min ,
\end{aligned}
$$

\footnotetext{
Keywords and phrases. Optimal control, sub-Riemannian geometry, differential-geometric methods, left-invariant problem, group of motions of a plane, rototranslations, conjugate time, cut time.

* Work supported by Russian Foundation for Basic Research, Project 09-01-00246-a, and by program of Presidium of Russian Academy of Sciences "Mathematical Control Theory".

${ }^{1}$ Program Systems Institute, Pereslavl-Zalessky, Russia. sachkov@sys.botik.ru
} 


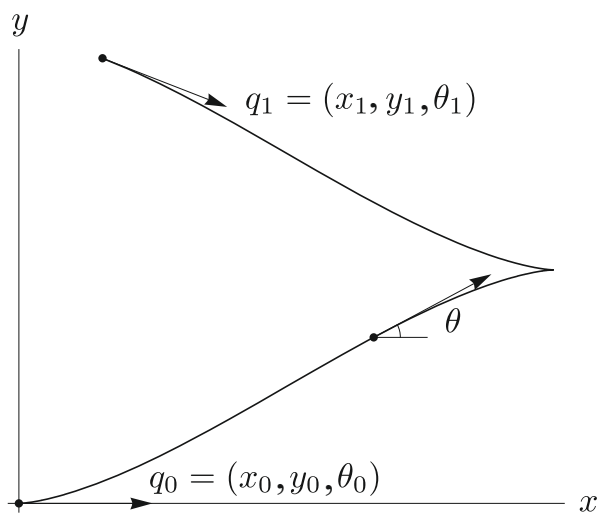

Figure 1. Problem statement.

or, equivalently,

$$
J=\frac{1}{2} \int_{0}^{t_{1}}\left(u_{1}^{2}+u_{2}^{2}\right) \mathrm{d} t \rightarrow \min .
$$

Problem (1.1)-(1.8) is a left-invariant sub-Riemannian problem on the group of motions of a plane $\mathrm{SE}(2)=$ $\mathbb{R}^{2} \ltimes \mathrm{SO}(2)$. This problem is closely connected to models of vision [5,10,11] and robotics [8]. On the other hand, this problem is important for understanding properties of the heat kernel of the diffusion equation on the group SE(2), see [4]. We expect that results of this work can be applied to these domains. On the other hand, problem (1.1)-(1.5) is the first completely studied sub-Riemannian problem without rotational symmetry; this problem has the local structure of generic contact sub-Riemannian problems as described in works $[1,6]$.

This is an immediate continuation of the previous work [9]. We use extensively the results obtained in that paper, and now we recall the most important of them.

The normal Hamiltonian system of Pontryagin Maximum Principle [12] becomes triangular in appropriate coordinates on cotangent bundle $T^{*} M$, and its vertical subsystem is the equation of mathematical pendulum:

$$
\begin{aligned}
& \dot{\gamma}=c, \quad \dot{c}=-\sin \gamma, \quad(\gamma, c) \in C \cong\left(2 S_{\gamma}^{1}\right) \times \mathbb{R}_{c} \\
& \dot{x}=\sin \frac{\gamma}{2} \cos \theta, \quad \dot{y}=\sin \frac{\gamma}{2} \sin \theta, \quad \dot{\theta}=-\cos \frac{\gamma}{2}
\end{aligned}
$$

The phase cylinder of pendulum (1.6) decomposes into invariant subsets according to values of the energy $E=c^{2} / 2-\cos \gamma$ :

$$
\begin{aligned}
& C=\bigcup_{i=1}^{5} C_{i}, \\
& C_{1}=\{\lambda \in C \mid E \in(-1,1)\}, \\
& C_{2}=\{\lambda \in C \mid E \in(1,+\infty)\}, \\
& C_{3}=\{\lambda \in C \mid E=1, c \neq 0\}, \\
& C_{4}=\{\lambda \in C \mid E=-1\}=\{(\gamma, c) \in C \mid \gamma=2 \pi n, c=0\}, \\
& C_{5}=\{\lambda \in C \mid E=1, c=0\}=\{(\gamma, c) \in C \mid \gamma=\pi+2 \pi n, c=0\} .
\end{aligned}
$$




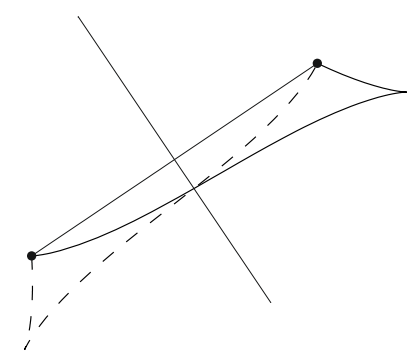

Figure 2. Action of $\varepsilon^{1}, \varepsilon^{2}$ on $\left(x_{t}, y_{t}\right)$.

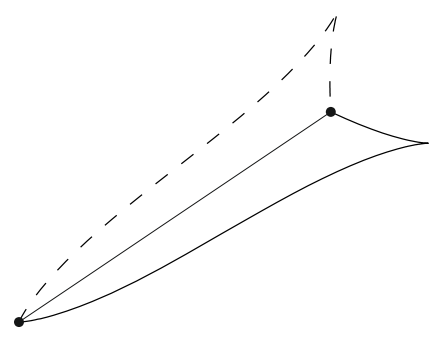

Figure 3. Action of $\varepsilon^{4}, \varepsilon^{7}$ on $\left(x_{t}, y_{t}\right)$.

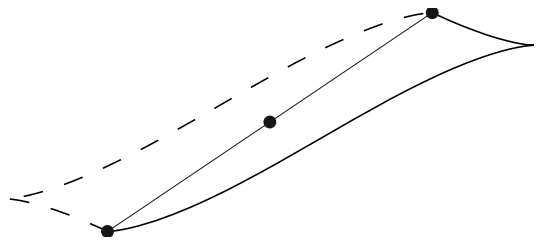

Figure 4. Action of $\varepsilon^{5}, \varepsilon^{6}$ on $\left(x_{t}, y_{t}\right)$.

In the subsets $C_{1}, C_{2}, C_{3}$ were introduced elliptic coordinates $(\varphi, k)$ that rectify the flow of the pendulum: $\varphi$ is the phase, and $k$ a reparameterized energy of pendulum (1.6):

$$
k=\sqrt{(E+1) / 2} \text { in } C_{1} \cup C_{3}, \quad k=\sqrt{2 /(E+1)} \text { in } C_{2} .
$$

The Hamiltonian system (1.6), (1.7) was integrated in Jacobi's functions [22]. The equation of pendulum (1.6) has a discrete group of symmetries $G=\left\{\operatorname{Id}, \varepsilon^{1}, \ldots, \varepsilon^{7}\right\}=\mathbb{Z}_{2} \times \mathbb{Z}_{2} \times \mathbb{Z}_{2}$ generated by reflections in the axes of coordinates $\gamma, c$, and translations $(\gamma, c) \mapsto(\gamma+2 \pi, c)$. Action of the group $G$ is naturally extended to extremal trajectories $\left(x_{t}, y_{t}\right)$, this action modulo rotations is represented at Figures $2-4$.

Reflections $\varepsilon^{i}$ are symmetries of the exponential mapping $\operatorname{Exp}: N=C \times \mathbb{R}_{+} \rightarrow M, \operatorname{Exp}(\lambda, t)=q_{t}$.

The main result of work [9] is an upper bound on cut time

$$
t_{\text {cut }}=\sup \left\{t_{1}>0 \mid q_{s} \text { is optimal for } s \in\left[0, t_{1}\right]\right\}
$$

along extremal trajectories $q_{s}$. It is based on the fact that a sub-Riemannian geodesic cannot be optimal after a Maxwell point, i.e., a point where two distinct geodesics of equal sub-Riemannian length meet one another. A natural idea is to look for Maxwell points corresponding to discrete symmetries of the exponential mapping. For each extremal trajectory $q_{s}=\operatorname{Exp}(\lambda, s)$, we described Maxwell times $t_{\varepsilon^{i}}^{n}(\lambda), i=1, \ldots, 7, n=1,2, \ldots$, corresponding to discrete symmetries $\varepsilon^{i}$. The following upper bound is the main result of work [9]:

$$
t_{\text {cut }}(\lambda) \leq \mathbf{t}(\lambda), \quad \lambda \in C,
$$

where $\mathbf{t}(\lambda)=\min \left(t_{\varepsilon^{i}}^{1}(\lambda)\right)$ is the first Maxwell time corresponding to the group of symmetries $G$. We recall the explicit definition of the function $\mathbf{t}(\lambda)$ below in equations (2.20)-(2.24).

In this work we continue the study of problem (1.1)-(1.5).

First we consider the local optimality of sub-Riemannian geodesics (Sect. 2). We show that extremal trajectories corresponding to oscillating pendulum (1.6) do not have conjugate points, thus they are locally optimal forever. In the case of rotating pendulum we prove that the first conjugate time is bounded from below and from above by the first Maxwell times $t_{\varepsilon^{2}}^{1}$ and $t_{\varepsilon^{5}}^{1}$ respectively. For critical values of energy of the pendulum, there are no conjugate points.

In Section 3 we study the global optimality of geodesics. We construct open dense subsets in the preimage and image of exponential mapping and prove that the exponential mapping transforms these domains diffeomorphically. As a consequence, we show that inequality (1.13) is in fact an equality. Moreover, we describe the optimal synthesis on the open dense subset of the state space.

In Section 4 we present plots of the sub-Riemannian caustic and spheres obtained in Mathematica [23].

In the subsequent work [19] we complete our study of problem (1.1)-(1.5). There we describe explicitly the Maxwell strata and cut locus, and characterize the optimal synthesis in this problem. For some special terminal points $q_{1}$, we provide explicit optimal solutions. 


\section{Conjugate points}

In this section we obtain bounds on conjugate time in the sub-Riemannian problem on $\mathrm{SE}(2)$, see Theorem 2.5.

\subsection{General facts}

First we recall some known facts from the theory of conjugate points in optimal control problems. For details see, e.g., $[2,3,20]$.

Consider an optimal control problem of the form

$$
\begin{aligned}
& \dot{q}=f(q, u), \quad q \in M, \quad u \in U \subset \mathbb{R}^{m}, \\
& q(0)=q_{0}, \quad q\left(t_{1}\right)=q_{1}, \quad t_{1} \text { fixed, } \\
& J=\int_{0}^{t_{1}} \varphi(q(t), u(t)) \mathrm{d} t \rightarrow \min ,
\end{aligned}
$$

where $M$ is a finite-dimensional analytic manifold, $f(q, u)$ and $\varphi(q, u)$ are respectively analytic in $(q, u)$ families of vector fields and functions on $M$ depending on the control parameter $u \in U$, and $U$ an open subset of $\mathbb{R}^{m}$. Admissible controls are $u(\cdot) \in L_{\infty}\left(\left[0, t_{1}\right], U\right)$, and admissible trajectories $q(\cdot)$ are Lipschitzian. Let

$$
h_{u}(\lambda)=\langle\lambda, f(q, u)\rangle-\varphi(q, u), \quad \lambda \in T^{*} M, \quad q=\pi(\lambda) \in M, \quad u \in U,
$$

be the normal Hamiltonian of PMP for the problem (2.1)-(2.3). Fix a triple $\left(\widetilde{u}(t), \lambda_{t}, q(t)\right)$ consisting of a normal extremal control $\widetilde{u}(t)$, the corresponding extremal $\lambda_{t}$, and the extremal trajectory $q(t)$ for the problem (2.1)-(2.3).

Let the following hypotheses hold:

(H1) For all $\lambda \in T^{*} M$ and $u \in U$, the quadratic form $\frac{\partial^{2} h_{u}}{\partial u^{2}}(\lambda)$ is negative definite.

(H2) For any $\lambda \in T^{*} M$, the function $u \mapsto h_{u}(\lambda), u \in U$, has a maximum point $\bar{u}(\lambda) \in U$ :

$$
h_{\bar{u}(\lambda)}(\lambda)=\max _{u \in U} h_{u}(\lambda), \quad \lambda \in T^{*} M .
$$

(H3) The extremal control $\widetilde{u}(\cdot)$ is a corank one critical point of the endpoint mapping.

(H4) All trajectories of the Hamiltonian vector field $\vec{H}(\lambda), \lambda \in T^{*} M$, are continued for $t \in[0,+\infty)$.

An instant $t_{*}>0$ is called a conjugate time (for the initial instant $t=0$ ) along the extremal $\lambda_{t}$ if the restriction of the second variation of the endpoint mapping to the kernel of its first variation is degenerate, see [3] for details. In this case the point $q\left(t_{*}\right)=\pi\left(\lambda_{t_{*}}\right)$ is called conjugate for the initial point $q_{0}$ along the extremal trajectory $q(\cdot)$.

Under hypotheses (H1)-(H4), we have the following:

(1) Normal extremal trajectories lose their local optimality (both strong and weak) at the first conjugate point, see [3].

(2) An instant $t>0$ is a conjugate time iff the exponential mapping $\operatorname{Exp}_{t}=\pi \circ \mathrm{e}^{t \vec{H}}$ is degenerate, see [2].

(3) Along each normal extremal trajectory, conjugate times are isolated one from another, see [20].

We will apply the following statement for the proof of absence of conjugate points via homotopy.

Proposition 2.1 (Cors. 2.2 and $2.3[18])$. Let $\left(u^{s}(t), \lambda_{t}^{s}\right), t \in[0,+\infty), s \in[0,1]$, be a continuous in parameter $s$ family of normal extremal pairs in the optimal control problem (2.1)-(2.3) satisfying hypotheses $(\mathbf{H 1})-(\mathbf{H} 4)$.

(1) Let $s \mapsto t_{1}^{s}$ be a continuous function, $s \in[0,1], t_{1}^{s} \in(0,+\infty)$. Assume that for any $s \in[0,1]$ the instant $t=t_{1}^{s}$ is not a conjugate time along the extremal $\lambda_{t}^{s}$.

If the extremal trajectory $q^{0}(t)=\pi\left(\lambda_{t}^{0}\right), t \in\left(0, t_{1}^{0}\right.$, does not contain conjugate points, then the extremal trajectory $q^{1}(t)=\pi\left(\lambda_{t}^{1}\right), t \in\left(0, t_{1}^{1}\right]$, also does not contain conjugate points. 
(2) Let for any $s \in[0,1)$ and $T>0$ the extremal $\lambda_{t}^{s}$ has no conjugate points for $t \in(0, T]$. Then for any $T>0$, the extremal $\lambda_{t}^{1}$ also has no conjugate points for $t \in(0, T]$.

One easily checks that the sub-Riemannian problem (1.1)-(1.5) satisfies all hypotheses (H1)-(H4), so the results cited in this subsection are applicable to this problem.

We denote the first conjugate time along an extremal trajectory $q(t)=\operatorname{Exp}(\lambda, t)$ as $t_{1}^{\text {conj }}(\lambda)$.

\subsection{Conjugate points for the case of oscillating pendulum}

In this section we assume that $\lambda \in C_{1}$ and prove that the corresponding extremal trajectories do not contain conjugate points, see Theorem 2.1.

Using the parameterization of extremal trajectories obtained in Section 3.3 [9], we compute explicitly Jacobian of the exponential mapping:

$$
\begin{aligned}
& J=\frac{\partial\left(x_{t}, y_{t}, \theta_{t}\right)}{\partial(t, \varphi, k)}=\frac{4}{k^{3}\left(1-k^{2}\right)\left(1-k^{2} \operatorname{sn}^{2} p \operatorname{sn}^{2} \tau\right)} J_{1}, \\
& p=t / 2, \quad \tau=\varphi+t / 2, \\
& J_{1}(\tau, p, k)=v_{1} \operatorname{sn}^{2} \tau+v_{2} \mathrm{cn}^{2} \tau, \\
& v_{1}=\left(1-k^{2}\right)(p-\mathrm{E}(p))\left(\mathrm{E}(p)-\left(1-k^{2}\right) p\right), \\
& v_{2}=(p-\mathrm{E}(p))\left(\mathrm{E}(p)-\left(1-k^{2}\right) p\right)+k^{2} \operatorname{cn} p \operatorname{dn} p\left(2 \mathrm{E}(p)+\left(k^{2}-2\right) p\right) \operatorname{sn} p \\
& \quad+k^{2}\left((\mathrm{E}(p)-p)\left(\mathrm{E}(p)-\left(1-k^{2}\right) p\right)-k^{2}\right) \operatorname{sn}^{2} p+k^{4} \operatorname{sn}^{4} p,
\end{aligned}
$$

so that $\operatorname{sgn} J=\operatorname{sgn} J_{1}$.

\subsubsection{Preliminary lemmas}

Lemma 2.1. For any $k \in(0,1)$ and $p>0$ we have $v_{1}(p, k)>0$.

Proof. The statement follows from the relations

$$
p-\mathrm{E}(p)=k^{2} \int_{0}^{p} \operatorname{sn}^{2} t \mathrm{~d} t>0, \quad \mathrm{E}(p)-\left(1-k^{2}\right) p=k^{2} \int_{0}^{p} \operatorname{cn}^{2} t \mathrm{~d} t>0 .
$$

Lemma 2.2. For any $k \in(0,1), n \in \mathbb{N}$, and $\tau \in \mathbb{R}$ we have $J_{1}(\tau, 2 K n, k)>0$.

Proof. If $p=2 K n, n \in \mathbb{N}$, then $v_{2}(p, k)=(p-\mathrm{E}(p))\left(\mathrm{E}(p)-\left(1-k^{2}\right) p\right)>0$ by inequalities (2.6).

By virtue of Lemma 2.1 and decomposition (2.5), we obtain the inequality $J_{1}(\tau, 2 K n, k)>0$.

Lemma 2.3. $\forall p_{1}>0, \quad \exists \widehat{k} \in(0,1), \quad \forall k \in(0, \widehat{k}), \quad \forall p \in\left(0, p_{1}\right), \quad \forall \tau \in \mathbb{R}, \quad J_{1}(\tau, p, k)>0$.

Proof. The statement of the lemma follows from the Taylor expansions:

$$
\begin{aligned}
J_{1} & =\frac{k^{4}}{16}\left(4 p^{2}-\sin ^{2} 2 p\right)+o\left(k^{4}\right), \quad k \rightarrow 0, \\
J_{1} & =\frac{1}{3} k^{4} p^{4}+o\left(k^{2}+p^{2}\right)^{4}, \quad k^{2}+p^{2} \rightarrow 0 .
\end{aligned}
$$

By contradiction, if the statement is not verified, then there exists a converging sequence $\left(\tau_{n}, p_{n}, k_{n}\right) \rightarrow\left(\tau_{0}, p_{0}, 0\right)$ such that $J\left(\tau_{n}, p_{n}, k_{n}\right) \leq 0$ for all $n \in N$. If $p_{0}>0$, then a standard calculus argument yields contradiction with (2.7). And if $p_{0}=0$, then similarly one obtains a contradiction with (2.8). 
2.2.2. Absence of conjugate points in $C_{1}$

Theorem 2.1. If $\lambda \in C_{1}$, then the extremal trajectory $q(t)=\operatorname{Exp}(\lambda, t), t>0$, does not contain conjugate points.

Proof. We choose any $\widehat{\lambda} \in C_{1}, \widehat{t}>0$, and prove that the extremal trajectory $\widehat{q}(t)=\operatorname{Exp}(\widehat{\lambda}, t)$ does not contain conjugate points for $t \in(0, \widehat{t}]$.

Find the elliptic coordinates $(\widehat{k}, \widehat{\varphi})$ corresponding to the covector $\widehat{\lambda} \in C_{1}$ according to Section 3.2 [9], and let $\widehat{p}=\widehat{t} / 2, \widehat{\tau}=\widehat{\varphi}+\widehat{p}$. Find $n \in \mathbb{N}$ such that $p_{1}=2 K(\widehat{k}) n>\widehat{p}$. Choose the following continuous curve in the plane $(k, p)$ :

$$
\left\{\left(k^{s}, p^{s}\right) \mid s \in[0,1]\right\}, \quad k^{s}=s \widehat{k}, \quad p^{s}=2 K\left(k^{s}\right) n,
$$

with the endpoints $\left(k^{0}, p^{0}\right)=(0, \pi n)$ and $\left(k^{1}, p^{1}\right)=(\widehat{k}, 2 K(\widehat{k}) n)$.

Consider the following family of extremal trajectories:

$$
\begin{aligned}
& \gamma^{s}=\left\{q^{s}(t)=\operatorname{Exp}\left(\varphi^{s}, k^{s}, t\right) \mid t \in\left[0, t^{s}\right]\right\}, \quad s \in[0,1], \\
& t^{s}=2 p^{s}, \quad \varphi^{s}=\widehat{\tau}-p^{s} .
\end{aligned}
$$

The endpoint $q^{s}\left(t^{s}\right)$ of each trajectory $\gamma^{s}, s \in[0,1]$, corresponds to the values of parameters $(\tau, p, k)=$ $\left(\widehat{\tau}, 2 K\left(k^{s}\right) n, k^{s}\right)$. Thus Lemma 2.2 implies that for any $s \in[0,1]$ the endpoint $q^{s}\left(t^{s}\right)$ is not a conjugate point.

Further, Lemma 2.3 states that

$$
\exists k_{0} \in(0, \widehat{k}) \quad \forall \tau \in \mathbb{R} \quad \forall p \in\left(0, p_{1}\right) \quad J(\tau, p, k)>0 .
$$

Denote $s_{0}=k_{0} / \widehat{k} \in(0,1)$, so that $k^{s_{0}}=k_{0}$. Condition (2.9) means that the extremal trajectory $\gamma^{s_{0}}$ does not contain conjugate points for all $t \in\left[0, t^{s_{0}}\right]$.

Then Proposition 2.1 yields that for any $s \in\left[s_{0}, 1\right]$, the extremal trajectory $q^{s}(t)$ does not contain conjugate points for all $t \in\left[0, t^{s}\right]$. In particular, the trajectory $\widehat{q}(t)=q^{1}(t), t \in(0, \widehat{t}]$, is free of conjugate points.

So we proved that extremal trajectories $q(t)=\operatorname{Exp}(\lambda, t)$ with $\lambda \in C_{1}$ (i.e., corresponding to oscillating pendulum) are locally optimal at any segment $\left[0, t_{1}\right], t_{1}>0$.

\subsection{Conjugate points for the case of rotating pendulum}

In this section we obtain bounds on conjugate points in the case $\lambda \in C_{2}$.

Using the formulas for extremal trajectories of Section 3.3 [9], we get:

$$
\begin{aligned}
& J=\frac{\partial\left(x_{t}, y_{t}, \theta_{t}\right)}{\partial(t, \varphi, k)}=-\frac{4 k}{\left(1-k^{2}\right)\left(1-k^{2} \operatorname{sn}^{2} p \operatorname{sn}^{2} \tau\right)} J_{2} \\
& p=t /(2 k), \quad \tau=\psi+t /(2 k)=(2 \varphi+t) /(2 k) \\
& J_{2}=\alpha \operatorname{sn}^{2} \tau+\beta \operatorname{cn}^{2} \tau \\
& \alpha=\left(1-k^{2}\right) \operatorname{sn} p \alpha_{1} \\
& \alpha_{1}=\operatorname{cn} p \operatorname{dn} p(p-2 \mathrm{E}(p))+\operatorname{sn} p\left(\operatorname{dn}^{2} p+\mathrm{E}(p)(p-\mathrm{E}(p))\right) \\
& \beta=f_{1}(p) \beta_{1}, \quad \beta_{1}=\operatorname{cn} p \mathrm{E}(p)-\operatorname{dn} p \operatorname{sn} p
\end{aligned}
$$

where $f_{1}(p, k)=\operatorname{cn} p(\mathrm{E}(p)-p)-\operatorname{dn} p \operatorname{sn} p$, see equation (5.12) [9].

\subsubsection{Preliminary lemmas}

Recall that we denoted the first positive root of the function $f_{1}(p)$ by $p_{1}^{1}(k)$, see Lemma $5.3[9]$.

Lemma 2.4. If $k \in(0,1)$ and $p=p_{1}^{1}(k)$, then $\alpha(p, k)>0$.

If additionally $\operatorname{sn} \tau \neq 0$, then $J_{2}>0$ and $J<0$. 
Proof. In terms of the auxiliary function

$$
\varphi(p, k)=\operatorname{sn} p \operatorname{dn} p-(2 \mathrm{E}(p)-p) \operatorname{cn} p,
$$

we have a decomposition

$$
\alpha_{1}=\operatorname{dn} p \varphi(p)+\operatorname{sn} p \mathrm{E}(p)(p-\mathrm{E}(p)) .
$$

Let $k \in(0,1)$ and $p=p_{1}^{1}(k)$. Then $f_{1}(p)=0$, i.e., $\operatorname{sn} p \operatorname{dn} p=\operatorname{cn} p(\mathrm{E}(p)-p)$. Thus $\varphi(p)=\operatorname{cn} p(\mathrm{E}(p)-$ $p)-(2 \mathrm{E}(p)-p) \operatorname{cn} p=-\mathrm{E}(p) \operatorname{cn} p$. By virtue of Corollary 5.1 [9], we have cn $p<0$, so $\varphi(p)>0$. Moreover, $\operatorname{sn} p>0$. Then decomposition (2.16) yields $\alpha_{1}(p)>0$, consequently, $\alpha(p)>0$.

If additionally $\operatorname{sn} \tau \neq 0$, then it is obvious that $J_{2}>0$ and $J<0$.

Lemma 2.5. $\exists \widehat{k} \in(0,1), \quad \forall k \in(0, \widehat{k}), \quad \forall p \in\left(0, p_{1}^{1}\right], \quad \alpha(p, k)>0$.

Proof. The statement of this lemma follows by the argument used in the proof of Lemma 2.3 from the Taylor expansions

$$
\begin{aligned}
& \alpha=\sin p(\sin p-p \cos p)+o(1), \quad k \rightarrow 0, \\
& \alpha=\frac{p^{4}}{3}+o\left(p^{2}+k^{2}\right)^{2}, \quad p^{2}+k^{2} \rightarrow 0 .
\end{aligned}
$$

Lemma 2.6. $\forall k \in(0,1), \quad \forall p \in(0,2 K], \quad \beta_{1}(p, k)<0$.

Proof. Since $\left(\beta_{1}(p) / \operatorname{cn} p\right)^{\prime}=-\left(1-k^{2}\right) \operatorname{sn}^{2} p / \mathrm{cn}^{2} p$, the function $\beta_{1}(p) / \operatorname{cn} p$ decreases at the segments $p \in[0, K)$ and $p \in(K, 2 K]$.

We have $\beta_{1}(0)=0$, thus $\beta_{1}(p) / \operatorname{cn} p<0$, so $\beta_{1}(p)<0$ for $p \in(0, K)$.

Further, $\beta_{1}(K)=-\sqrt{1-k^{2}}<0$.

Since $\beta_{1}(p) / \operatorname{cn} p \rightarrow+\infty$ as $p \rightarrow K+0$, and $\beta_{1}(2 K) / \operatorname{cn}(2 K)=\mathrm{E}(2 K)>0$, we have $\beta_{1}(p) / \operatorname{cn} p>0$, so $\beta_{1}(p)<0$ for $p \in(K, 2 K]$.

Lemma 2.7. Let $k \in(0,1)$.

(1) Let $\operatorname{sn} \tau=0$. Then $J_{2}(\tau, p, k)>0$ for $p \in\left(0, p_{1}^{1}\right)$, and $J_{2}(\tau, p, k)=0$ for $p=p_{1}^{1}$.

(2) Let $\operatorname{sn} \tau \neq 0$. Then $J_{2}(\tau, p, k)>0$ for $p \in\left(0, p_{1}^{1}\right]$.

Proof. If $p \in\left(0, p_{1}^{1}\right)$, then $f_{1}(p, k)<0$ (Cor. 5.1 [9]), and $\beta_{1}(p, k)<0$ (Lem. 2.6), thus $\beta(p, k)=$ $f_{1}(p, k) \beta_{1}(p, k)>0$.

(1) Let $\operatorname{sn} \tau=0$. If $p \in\left(0, p_{1}^{1}\right)$, then $J_{2}(\tau, p, k)=\beta(p, k)>0$. And if $p=p_{1}^{1}$, then $f_{1}(p, k)=0$, thus $J_{2}(\tau, p, k)=\beta(p, k)=0$.

(2) Let $\operatorname{sn} \tau \neq 0$.

(2.a) We prove that the function $\varphi(p)$ given by (2.15) satisfies the inequality

$$
\varphi(p)>0 \quad \forall p \in(0, K] .
$$

First, $\varphi(p)=p^{3} / 3+o\left(p^{3}\right)>0$ as $p \rightarrow+0$. Second,

$$
(\varphi(p) / \operatorname{cn} p)^{\prime}=\operatorname{dn}^{2} p \operatorname{sn}^{2} p / \operatorname{cn}^{2} p>0 \quad \forall p \in(0, K) .
$$

Thus $\varphi(p)>0$ for $p \in(0, K)$. And if $p=K$, then $\varphi(p)=\sqrt{1-k^{2}}>0$.

(2.b) By virtue of the decomposition $\varphi(p)=-f_{1}(p)-\mathrm{E}(p) \operatorname{cn} p$, we get the inequality $\varphi(p)>0$ for all $p \in\left(K, p_{1}^{1}\right]$. We proved that

$$
\varphi(p)>0 \quad \forall p \in\left(0, p_{1}^{1}\right] .
$$

(2.c) In view of (2.16), we obtain that $\alpha_{1}(p)>0$ for $p \in\left(0, p_{1}^{1}\right]$. Then equation (2.12) yields $\alpha(p)>0$ for $p \in\left(0, p_{1}^{1}\right]$. Finally, equation (2.11) gives $J_{2}>0$ for $p \in\left(0, p_{1}^{1}\right]$. 
Lemma 2.8. $\forall z \in(0,1], \quad \exists \widehat{k} \in(0,1), \quad \forall k \in(0, \widehat{k}), \quad \forall p \in\left(0, p_{1}^{1}\right], \quad$ we have $J_{2}(z, p, k)>0$.

Proof. Fix any $z \in(0,1]$. By Lemma 2.5,

$$
\exists \widehat{k} \in(0,1), \quad \forall k \in(0, \widehat{k}), \quad \forall p \in\left(0, p_{1}^{1}\right], \quad \alpha(p, k)>0 .
$$

But if $p \in\left(0, p_{1}^{1}\right]$, then $p \in(0,2 K]$, thus $\beta_{1}(p, k)<0$ by Lemma 2.6, so $\beta(p, k)>0$ by Corollary 5.1 [9].

Then the inequalities $\alpha(p, k)>0, \beta(p, k)>0$ imply the required inequality $J_{2}(\tau, p, k)>0$.

\subsubsection{Conjugate points in $C_{2}$}

First we obtain a lower bound on the first conjugate time. It will play a crucial role in the subsequent analysis of the global structure of the exponential mapping in Section 3 and in the subsequent work [19].

Theorem 2.2. If $\lambda \in C_{2}$, then $t_{1}^{\mathrm{conj}}(\lambda) \geq 2 k p_{1}^{1}(k)$.

Proof. Given any $\lambda \in C_{2}$, compute the corresponding elliptic coordinates $(\varphi, k)$. If additionally we have $t>0$, find the corresponding parameters $p=t /(2 k), \tau=\varphi / k+t /(2 k)$ and denote $z=\operatorname{sn}^{2} \tau$.

We should prove that for any $\lambda \in C_{2}$ the interval $t \in\left(0,2 k p_{1}^{1}(k)\right)$ does not contain conjugate times for the extremal trajectory $q(t)=\operatorname{Exp}(\lambda, t)$.

Take any $\lambda^{1} \in C_{2}$ and denote the corresponding elliptic coordinates $\left(\varphi^{1}, k^{1}\right)$. For $t^{1}=2 k^{1} p_{1}^{1}\left(k^{1}\right)$ we denote the corresponding parameters $p^{1}, \tau^{1}, z^{1}$. In order to prove that the extremal trajectory $q^{1}(t)=\operatorname{Exp}\left(\lambda^{1}, t\right)$ does not have conjugate points at the interval $t \in\left(0, t^{1}\right)$, we show that

$$
p \in\left(0, p^{1}\right) \quad \Rightarrow \quad J_{2}\left(z^{1}, p, k^{1}\right)>0 \quad \Rightarrow \quad J\left(z^{1}, p, k^{1}\right)<0 .
$$

(1) Assume first that $z^{1}=\operatorname{sn}^{2}\left(\tau^{1}, k^{1}\right) \neq 0$, i.e., $z^{1} \in(0,1]$. We prove that in this case

$$
p \in\left(0, p^{1}\right] \quad \Rightarrow \quad J\left(z^{1}, p, k^{1}\right)<0 .
$$

Consider the following continuous curve in the space $(z, p, k)$ :

$$
\left\{\left(z^{1}, p^{s}, k^{s}\right) \mid s \in(0,1]\right\}, \quad k^{s}=s k^{1}, \quad p^{s}=p_{1}^{1}\left(k^{s}\right) .
$$

The corresponding curve in the space $(\tau, p, k)$ is

$$
\left\{\left(\tau^{s}, p^{s}, k^{s}\right) \mid s \in(0,1]\right\}, \quad \tau^{s}=F\left(\operatorname{am}\left(\tau^{1}, k^{1}\right), k^{s}\right),
$$

and in the space $(t, \varphi, k)$ is

$$
\left\{\left(t^{s}, \varphi^{s}, k^{s}\right) \mid s \in(0,1]\right\}, \quad t^{s}=2 k^{s} p^{s}, \quad \varphi^{s}=\left(\tau^{s}-p^{s}\right) k^{s} .
$$

Let $\lambda^{s}=\left(\varphi^{s}, k^{s}\right), s \in(0,1]$, be the corresponding curve in $C_{2}$, and consider the continuous one-parameter family of extremal trajectories

$$
q^{s}(t)=\operatorname{Exp}\left(\lambda^{s}, t\right), \quad t \in\left[0, t^{s}\right], \quad s \in(0,1] .
$$

For any $s \in(0,1]$, if $t=t^{s}$, then by Lemma 2.4 we have $J_{2}\left(z^{1}, p_{1}^{1}\left(k^{s}\right), k^{s}\right)<0$, i.e., the terminal instant $t=t^{s}$ is not a conjugate time along the extremal trajectory $q^{s}(t)$.

Further, by Lemma 2.8, for $z^{1} \in(0,1]$

$$
\exists \widehat{k} \in(0,1), \quad \forall k \in(0, \widehat{k}), \quad \forall p \in\left(0, p_{1}^{1}(k)\right], \quad J_{2}\left(z^{1}, p, k\right)>0 .
$$


Consequently, there exists $s_{0} \in(0,1)$ such that the whole trajectory $q^{s_{0}}(t), t \in\left(0, t^{s_{0}}\right]$, is free of conjugate points.

Then Proposition 2.1 implies that the trajectory $q^{1}(t), t \in\left(0, t^{1}\right]$, also does not contain conjugate points.

We proved that if $z^{1} \neq 0$, then the trajectory $q^{1}(t)=\operatorname{Exp}\left(\lambda^{1}, t\right), t \in\left(0, t^{1}\right]$, does not have conjugate points.

(2) Now consider the case $z^{1}=\operatorname{sn}^{2}\left(\tau^{1}, k^{1}\right)=0$. Then Lemma 2.7 states that the terminal instant $t=$ $2 k^{1} p_{1}^{1}\left(k^{1}\right)$ is a conjugate point. We prove that all the less instants are not conjugate.

Since conjugate points are isolated one from another at each extremal trajectory, there exists $p<p_{1}^{1}\left(k^{1}\right)$ arbitrarily close to $p_{1}^{1}\left(k^{1}\right)$ such that the corresponding time $t=2 k^{1} p$ is not conjugate.

Consider the continuous curve in the space $(z, p, k)$ :

$$
\left\{\left(z_{s}, p, k^{1}\right) \mid s \in[0, \varepsilon)\right\}, \quad z_{s}=s z^{1} .
$$

By item (1) of this proof, there exists $\varepsilon>0$ such that for any $s \in(0, \varepsilon)$ the extremal trajectory $q^{s}(t), t \in\left(0, t^{s}\right]$, $t^{s}=2 k^{1} p$, does not have conjugate points. By Proposition 2.1, for $s=0$ the initial extremal trajectory $q^{0}(t)$, $t \in\left(0, t^{0}\right]$, also does not contain conjugate points. The endpoint $t^{0}=2 k^{1} p$ can be chosen arbitrarily close to $t^{1}=2 k^{2} p_{1}^{1}\left(k^{1}\right)$, so the initial extremal trajectory does not have conjugate points for $t \in\left(0, t^{1}\right)$.

Now we obtain the final result on the first conjugate time in the domain $C_{2}-$ the following two-side bound.

Theorem 2.3. If $\lambda \in C_{2}$, then

$$
2 k p_{1}^{1}(k) \leq t_{1}^{\mathrm{conj}}(\lambda) \leq 4 k K(k) .
$$

Proof. We proved in Theorem 2.2 that $2 k p_{1}^{1}(k) \leq t_{1}^{\text {conj }}(\lambda)$; moreover, if $t \in\left(0,2 k p_{1}^{1}\right)$, then $J<0$.

Let $t=4 k K$, then $p=2 K$, thus $\alpha=0, f_{1}=p-\mathrm{E}(p)>0, \beta_{1}=-\mathrm{E}(p)<0$, so

$$
J=-\frac{4 k}{1-k^{2}} J_{2}=\frac{4 k}{1-k^{2}} \mathrm{cn}^{2} \tau \mathrm{E}(p)(p-\mathrm{E}(p)) \geq 0 .
$$

It follows that for any $\lambda \in C_{2}$ the function $t \mapsto J$ has a root at the segment $t \in\left[2 k p_{1}^{1}, 4 k K\right]$. Consequently, also the first root $t_{1}^{\mathrm{conj}} \in\left[2 k p_{1}^{1}, 4 k K\right]$.

One can show that the bound (2.17) can be a little bit improved. The precise bound on the first conjugate time is

$$
2 k p_{1}^{1}(k) \leq t_{1}^{\operatorname{conj}}(\lambda) \leq \gamma(k)=\min \left(4 k K, 2 k p_{1}^{\alpha_{1}}(k)\right),
$$

where $p=p_{1}^{\alpha_{1}}(k)$ is the first positive root of the equation $\alpha_{1}(p)=0$, and the function $\alpha_{1}$ is given by equation (2.13). One can show that $\gamma(k)=4 k K$ for $k \in\left(0, k_{0}\right]$ and $\gamma(k)=2 k p_{1}^{\alpha_{1}}(k)$ for $\left[k_{0}, 1\right)$, where $k_{0} \approx 0.909$ is the unique root of the equation $2 E(k)-K(k)=0$, see Proposition 11.5 [17]. Thus for $k \in\left(k_{0}, 1\right)$ the bound (2.17) is not exact and can be replaced by the following exact one:

$$
2 k p_{1}^{1}(k) \leq t_{1}^{\operatorname{conj}}(\lambda) \leq 2 k p_{1}^{\alpha_{1}}(k), \quad k \in\left(k_{0}, 1\right) .
$$

The bound (2.17) is illustrated at Figures 5 and 6; and the bound (2.19) - at Figure 7. The exact bounds (2.18) are plotted at Figure 8 .

Proposition 2.2. Let $\lambda \in C_{2}$ and $\tau=\left(2 \varphi+2 k p_{1}^{1}\right) /(2 k)$.

(1) If $\operatorname{sn} \tau=0$, then $t_{1}^{\text {conj }}(\lambda)=2 k p_{1}^{1}$.

(2) If $\operatorname{sn} \tau \neq 0$, then $t_{1}^{\text {conj }}(\lambda) \in\left(2 k p_{1}^{1}, 4 k K\right]$.

Proof. Notice first that by Theorem 2.2, the interval $\left(0,2 k p_{1}^{1}\right)$ does not contain conjugate times. Then items (1), (2) of this proposition follow directly from the corresponding items of Lemma 2.7, and from Theorem 2.3. 
$t$

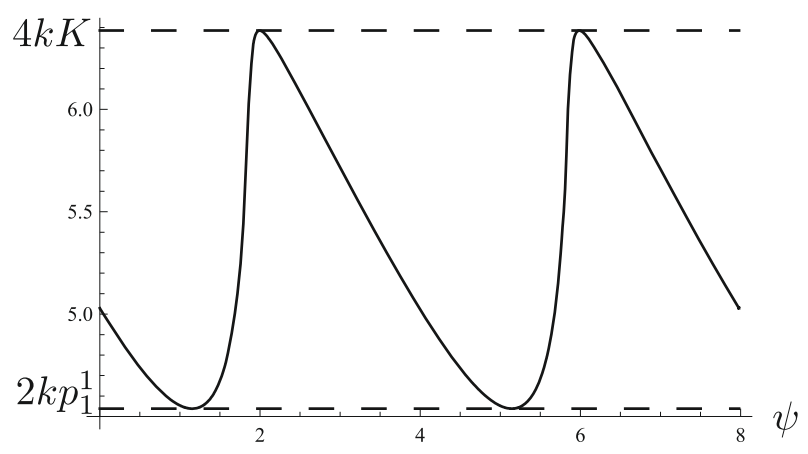

Figure 5. Plot of $t_{1}^{\text {conj }}(\psi, k)$, $k=0.8<k_{0}$.

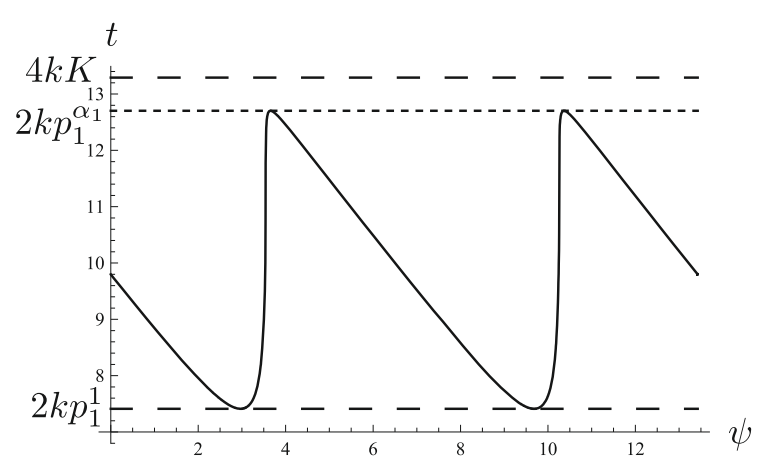

Figure 7. Plot of $t_{1}^{\text {conj }}(\psi, k)$, $k=0.99>k_{0}$.

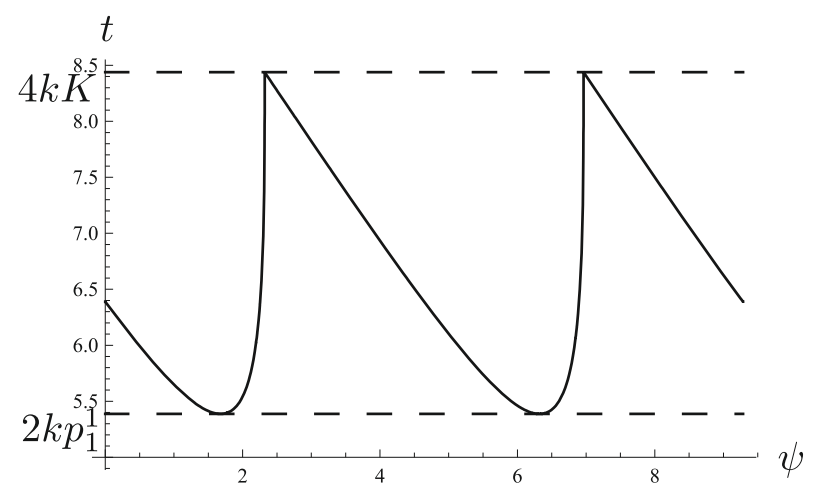

Figure 6. Plot of $t_{1}^{\text {conj }}(\psi, k)$, $k=k_{0}$.

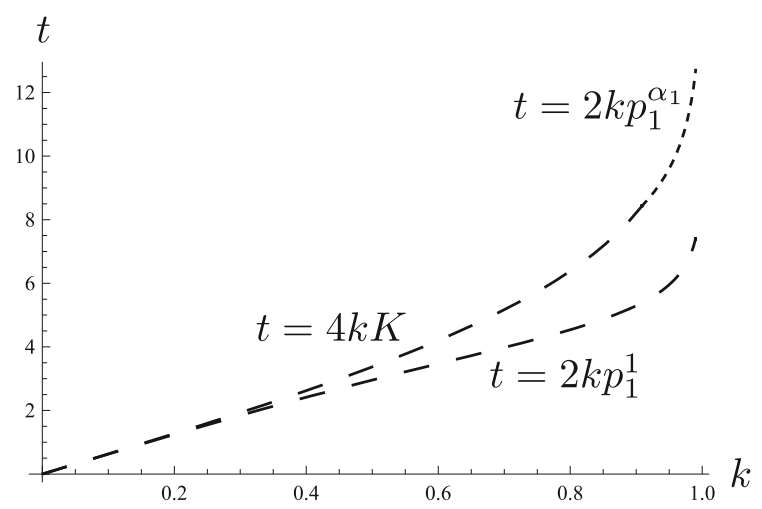

Figure 8. Bounds of $t_{1}^{\mathrm{conj}}(\psi, k)$.

\subsection{Conjugate points for the cases of critical energy of pendulum}

The subset $C_{3} \cup C_{4} \cup C_{5}$ of the cylinder $C$ is the boundary of the domain $C_{1}$, see equations (1.8)-(1.12) above, and Figure 2 in [9]. Thus absence of conjugate points for the corresponding extremal trajectories follows by limit passage from $C_{1}$.

Theorem 2.4. If $\lambda \in C_{3} \cup C_{4} \cup C_{5}$, then the corresponding extremal trajectory $q(t)=\operatorname{Exp}(\lambda, t)$ does not have conjugate points for $t>0$.

Proof. For any $\lambda \in C_{3} \cup C_{4} \cup C_{5}$, there exists a continuous curve $\lambda^{s}, s \in[0,1]$, such that $\lambda^{s} \in C_{1}$ for $s \in[0,1)$ and $\lambda^{1}=\lambda$. By Theorem 2.1, the trajectories $q^{s}(t)=\operatorname{Exp}\left(\lambda^{s}, t\right), t>0$, are free of conjugate points. Then Proposition 2.1 implies the same for the trajectory $q^{1}(t)=q(t)$.

\subsection{General bound of conjugate points}

We collect the bounds on the first conjugate time obtained in the previous subsections. Moreover, we conclude that the first conjugate time $t_{1}^{\text {conj }}$ admits the lower bound by the function $\mathbf{t}: C \rightarrow(0,+\infty]$ on the phase cylinder 
of pendulum $\left(2 S_{\gamma}^{1}\right) \times \mathbb{R}_{c}=C=\sqcup_{i=1}^{5} C_{i}$ introduced in [9]:

$$
\begin{aligned}
& \lambda \in C_{1} \quad \Rightarrow \quad \mathbf{t}(\lambda)=2 K(k), \\
& \lambda \in C_{2} \quad \Rightarrow \quad \mathbf{t}(\lambda)=2 k p_{1}^{1}(k), \\
& \lambda \in C_{3} \quad \Rightarrow \quad \mathbf{t}(\lambda)=+\infty, \\
& \lambda \in C_{4} \quad \Rightarrow \quad \mathbf{t}(\lambda)=\pi, \\
& \lambda \in C_{5} \quad \Rightarrow \quad \mathbf{t}(\lambda)=+\infty .
\end{aligned}
$$

Theorem 2.5. (1) If $\lambda \in C_{1} \cup C_{3} \cup C_{4} \cup C_{5}$, then $t_{1}^{\text {conj }}(\lambda)=+\infty$.

(2) If $\lambda \in C_{2}$, then $t_{1}^{\text {conj }}(\lambda) \in\left[2 k p_{1}^{1}, 4 k K\right]$.

(3) Consequently, $t_{1}^{\text {conj }}(\lambda) \geq \mathbf{t}(\lambda)$ for all $\lambda \in C$.

\section{EXPONENTIAL MAPPING OF OPEN STRATAS AND CUT TIME}

In this section we show that there exist open dense domains $\widetilde{N} \subset N, \widetilde{M} \subset M$ transformed diffeomorphically by the exponential mapping. As a consequence, we prove that $t_{\text {cut }}(\lambda)=\mathbf{t}(\lambda)$ for any $\lambda \in C$, and describe the optimal synthesis on $\widetilde{M}$.

\subsection{Decompositions in preimage and image of exponential mapping}

Denote $\widehat{M}=M \backslash\left\{q_{0}\right\}$. For any point $q \in \widehat{M}$ there exists an optimal trajectory $q(s)=\operatorname{Exp}(\lambda, s)$ such that $q(t)=q,(\lambda, t) \in N$. Thus the mapping $\operatorname{Exp}: N \rightarrow \widehat{M}$ is surjective. By Theorem 5.4 [9], the optimal instant $t$ satisfies the inequality $t \leq \mathbf{t}(\lambda)$. So the restriction

$$
\begin{aligned}
& \operatorname{Exp}: \widehat{N} \rightarrow \widehat{M}, \\
& \widehat{N}=\{(\lambda, t) \in N \mid t \leq \mathbf{t}(\lambda)\},
\end{aligned}
$$

is surjective as well.

\subsubsection{Decomposition in $\widehat{N}$}

Now we select open dense subsets of $\widehat{N}$ such that restriction of Exp to these subsets will turn out to be a diffeomorphism. Let

$$
\begin{aligned}
& N_{i}=C_{i} \times \mathbb{R}_{+}, \quad i=1, \ldots, 5, \\
& \widetilde{N}=\left\{(\lambda, t) \in \cup_{i=1}^{3} N_{i} \mid t<\mathbf{t}(\lambda), \text { sn } \tau \operatorname{cn} \tau \neq 0\right\}, \\
& N^{\prime}=\left\{(\lambda, t) \in \cup_{i=1}^{3} N_{i} \mid t=\mathbf{t}(\lambda) \text { or } \operatorname{sn} \tau \operatorname{cn} \tau=0\right\} \cup \widehat{N}_{4} \cup N_{5}, \\
& \widehat{N}_{4}=\widehat{N} \cap N_{4} .
\end{aligned}
$$

We have the obvious decomposition $\widehat{N}=\widetilde{N} \sqcup N^{\prime}$ (we denote by $\sqcup$ the union of mutually non-intersecting sets).

There hold the following implications, see [9]:

$$
\begin{aligned}
& (\lambda, t) \in N_{1} \quad \Rightarrow \quad \mathbf{t}(\lambda)=2 K, \tau \in \mathbb{R} /(4 K \mathbb{Z}), \\
& (\lambda, t) \in N_{2} \quad \Rightarrow \quad \mathbf{t}(\lambda)=2 k p_{1}^{1}, \tau \in \mathbb{R} /(4 K \mathbb{Z}), \\
& (\lambda, t) \in N_{3} \quad \Rightarrow \quad \mathbf{t}(\lambda)=+\infty, \tau \in \mathbb{R} .
\end{aligned}
$$


TABle 1. Definition of domains $D_{i}$.

\begin{tabular}{|c|c|c|c|c|c|c|c|c|}
\hline$D_{i}$ & $D_{1}$ & $D_{2}$ & $D_{3}$ & $D_{4}$ & $D_{5}$ & $D_{6}$ & $D_{7}$ & $D_{8}$ \\
\hline$\lambda$ & $C_{1}^{0}$ & $C_{1}^{0}$ & $C_{1}^{0}$ & $C_{1}^{0}$ & $C_{1}^{1}$ & $C_{1}^{1}$ & $C_{1}^{1}$ & $C_{1}^{1}$ \\
$\tau$ & $(3 K, 4 K)$ & $(0, K)$ & $(K, 2 K)$ & $(2 K, 3 K)$ & $(-K, 0)$ & $(0, K)$ & $(K, 2 K)$ & $(2 K, 3 K)$ \\
$p$ & $(0, K)$ & $(0, K)$ & $(0, K)$ & $(0, K)$ & $(0, K)$ & $(0, K)$ & $(0, K)$ & $(0, K)$ \\
\hline$\lambda$ & $C_{2}^{+}$ & $C_{2}^{+}$ & $C_{2}^{-}$ & $C_{2}^{-}$ & $C_{2}^{+}$ & $C_{2}^{+}$ & $C_{2}^{-}$ & $C_{2}^{-}$ \\
$\tau$ & $(-K, 0)$ & $(0, K)$ & $(-K, 0)$ & $(0, K)$ & $(K, 2 K)$ & $(2 K, 3 K)$ & $(-3 K,-2 K)$ & $(-2 K,-K)$ \\
$p$ & $\left(0, p_{1}^{1}\right)$ & $\left(0, p_{1}^{1}\right)$ & $\left(0, p_{1}^{1}\right)$ & $\left(0, p_{1}^{1}\right)$ & $\left(0, p_{1}^{1}\right)$ & $\left(0, p_{1}^{1}\right)$ & $\left(0, p_{1}^{1}\right)$ & $\left(0, p_{1}^{1}\right)$ \\
\hline$\lambda$ & $C_{3}^{0+}$ & $C_{3}^{0+}$ & $C_{3}^{0-}$ & $C_{3}^{0-}$ & $C_{3}^{1+}$ & $C_{3}^{1+}$ & $C_{3}^{1-}$ & $C_{3}^{1-}$ \\
$\tau$ & $(-\infty, 0)$ & $(0,+\infty)$ & $(-\infty, 0)$ & $(0,+\infty)$ & $(-\infty, 0)$ & $(0,+\infty)$ & $(-\infty, 0)$ & $(0,+\infty)$ \\
$p$ & $(0,+\infty)$ & $(0,+\infty)$ & $(0,+\infty)$ & $(0,+\infty)$ & $(0,+\infty)$ & $(0,+\infty)$ & $(0,+\infty)$ & $(0,+\infty)$ \\
\hline
\end{tabular}

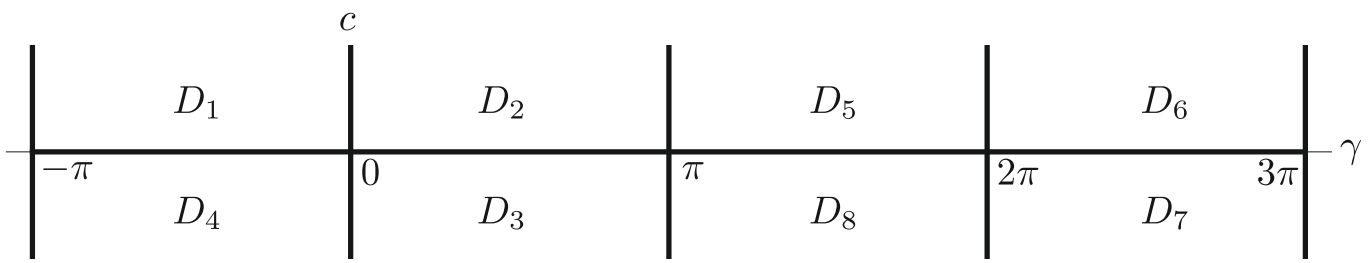

Figure 9. Projections of domains $D_{i}$ to the phase cylinder of pendulum $\left(2 S_{\gamma}^{1}\right) \times \mathbb{R}_{c}^{1}$.

Consequently, there holds the following decomposition:

$$
\widetilde{N}=\sqcup_{i=1}^{8} D_{i}
$$

where the sets $D_{i}, i=1, \ldots, 8$, are defined by Table 1 .

Table 1 should be read by columns. For example, the first column means that

$$
\begin{aligned}
& D_{1}=\left(D_{1} \cap N_{1}\right) \sqcup\left(D_{1} \cap N_{2}\right) \sqcup\left(D_{1} \cap N_{3}\right), \\
& D_{1} \cap N_{1}=\left\{(\tau, p, k) \in N_{1} \mid \lambda \in C_{1}^{0}, \tau \in(3 K, 4 K), p \in(0, K), k \in(0,1)\right\}, \\
& D_{1} \cap N_{2}=\left\{(\tau, p, k) \in N_{2} \mid \lambda \in C_{2}^{+}, \tau \in(-K, 0), p \in\left(0, p_{1}^{1}\right), k \in(0,1)\right\}, \\
& D_{1} \cap N_{3}=\left\{(\tau, p, k) \in N_{3} \mid \lambda \in C_{3}^{0+}, \tau \in(-\infty, 0), p \in(0,+\infty), k=1\right\} .
\end{aligned}
$$

Projections of the sets $D_{i}$ to the phase cylinder of the pendulum $(\gamma, c)$ are shown in Figure 9.

Lemma 3.1. Each set $D_{i}, i=1, \ldots, 8$, is homeomorphic to $\mathbb{R}^{3}$.

Proof. We prove the statement only for the set $D_{2}$ since all other sets $D_{i}$ can be defined in the coordinates $(\tau, p, k)$ by the same inequalities as $D_{2}$ by a shift of origin in elliptic coordinate $\varphi$. Taking into account Table 1 
TABle 2. Definition of domains $M_{i}$.

\begin{tabular}{|c|c|c|c|c|c|c|c|c|}
\hline$M_{i}$ & $M_{1}$ & $M_{2}$ & $M_{3}$ & $M_{4}$ & $M_{5}$ & $M_{6}$ & $M_{7}$ & $M_{8}$ \\
\hline $\operatorname{sgn}(\sin \theta)$ & - & - & - & - & + & + & + & + \\
\hline $\operatorname{sgn}\left(R_{1}\right)$ & + & + & - & - & - & - & + & + \\
\hline $\operatorname{sgn}\left(R_{2}\right)$ & + & - & - & + & + & - & - & + \\
\hline
\end{tabular}

and equations (2.4), (2.10), we get:

$$
\begin{aligned}
& D_{2}=\left(D_{2} \cap N_{1}\right) \sqcup\left(D_{2} \cap N_{2}\right) \sqcup\left(D_{2} \cap N_{3}\right), \\
& D_{2} \cap N_{1}=\left\{(\lambda, t) \in N_{1} \mid \lambda \in C_{1}^{0}, k \in(0,1), t \in(0,2 K), \varphi \in(-t,-t+2 K)\right\}, \\
& D_{2} \cap N_{2}=\left\{(\lambda, t) \in N_{1} \mid \lambda \in C_{2}^{+}, k \in(0,1), t \in\left(0,2 k p_{1}^{1}\right), \varphi \in(-t,-t+2 k K)\right\}, \\
& D_{2} \cap N_{3}=\left\{(\lambda, t) \in N_{1} \mid \lambda \in C_{3}^{0+}, k=1, t \in(0,+\infty), \varphi \in(-t,+\infty)\right\} .
\end{aligned}
$$

As shown in [17], one can choose regular system of coordinates $\left(k_{1}, \varphi, t\right)$ on the set $D_{2}$, where

$$
k_{1}=k \text { for } \lambda \in C_{1} ; \quad k_{1}=1 / k \text { for } \lambda \in C_{2} ; \quad k_{1}=1 \text { for } \lambda \in C_{3} .
$$

In this system of coordinates

$$
D_{2}=\left\{\nu=\left(k_{1}, \varphi, t\right) \mid k_{1} \in(0,+\infty), t \in\left(0, t_{1}\left(k_{1}\right), \varphi \in\left(-t,-t+t_{2}\left(k_{2}\right)\right)\right\}\right.
$$

where $t_{1}\left(k_{1}\right)=2 K\left(k_{1}\right)$ for $k_{1} \in(0,1), t_{1}\left(k_{1}\right)=+\infty$ for $k_{1}=1, t_{1}\left(k_{1}\right)=\left(2 / k_{1}\right) p_{1}^{1}\left(1 / k_{1}\right)$ for $k_{1} \in(1,+\infty)$; and $t_{2}\left(k_{1}\right)=2 K\left(k_{1}\right)$ for $k_{1} \in(0,1), t_{2}\left(k_{1}\right)=+\infty$ for $k_{1}=1, t_{2}\left(k_{1}\right)=\left(2 / k_{1}\right) K\left(1 / k_{1}\right)$ for $k_{1} \in(1,+\infty)$. The both functions $t_{i}:(0,+\infty) \rightarrow(0,+\infty], i=1,2$, are continuous. Thus representation (3.2) implies that the domain $D_{2}$ is homeomorphic to $\mathbb{R}^{3}$.

Consequently, all domains $D_{i}$ are open, connected, and simply connected. These domains are schematically represented in the left-hand side of Figure 10.

\subsubsection{Decomposition in $\widehat{M}$}

The state space of the problem admits a decomposition of the form

$$
\begin{aligned}
& M=\widetilde{M} \sqcup M^{\prime}, \\
& \widetilde{M}=\left\{q \in M \mid R_{1}(q) R_{2}(q) \sin \theta \neq 0\right\}, \\
& M^{\prime}=\left\{q \in M \mid R_{1}(q) R_{2}(q) \sin \theta=0\right\},
\end{aligned}
$$

where

Further,

$$
R_{1}=y \cos \frac{\theta}{2}-x \sin \frac{\theta}{2}, \quad R_{2}=x \cos \frac{\theta}{2}+y \sin \frac{\theta}{2}
$$

$$
\widetilde{M}=\sqcup_{i=1}^{8} M_{i}
$$

where each of the sets $M_{i}$ is characterized by constant signs of the functions $\sin \theta, R_{1}, R_{2}$ described in Table 2 .

For example, $M_{1}=\left\{q \in M \mid \sin \theta<0, R_{1}>0, R_{2}>0\right\}$. The numeration of the sets $M_{i}$ is chosen so that it correspond to numeration of the sets $N_{i}$ (we prove below in Thm. 3.1 that each mapping $\operatorname{Exp}: N_{i} \rightarrow M_{i}$ is a diffeomorphism). It is obvious that all the sets $M_{i}$ are diffeomorphic to $\mathbb{R}^{3}$.

All the domains $M_{i}$ are contained in the set $\{q \in M \mid \theta \neq 0\}$. At this set $\theta$ is a single-valued function, and we choose the branch $\theta \in(0,2 \pi)$. Thus in the sequel we assume that $\theta \in(0,2 \pi)$ on the sets $M_{i}$. 

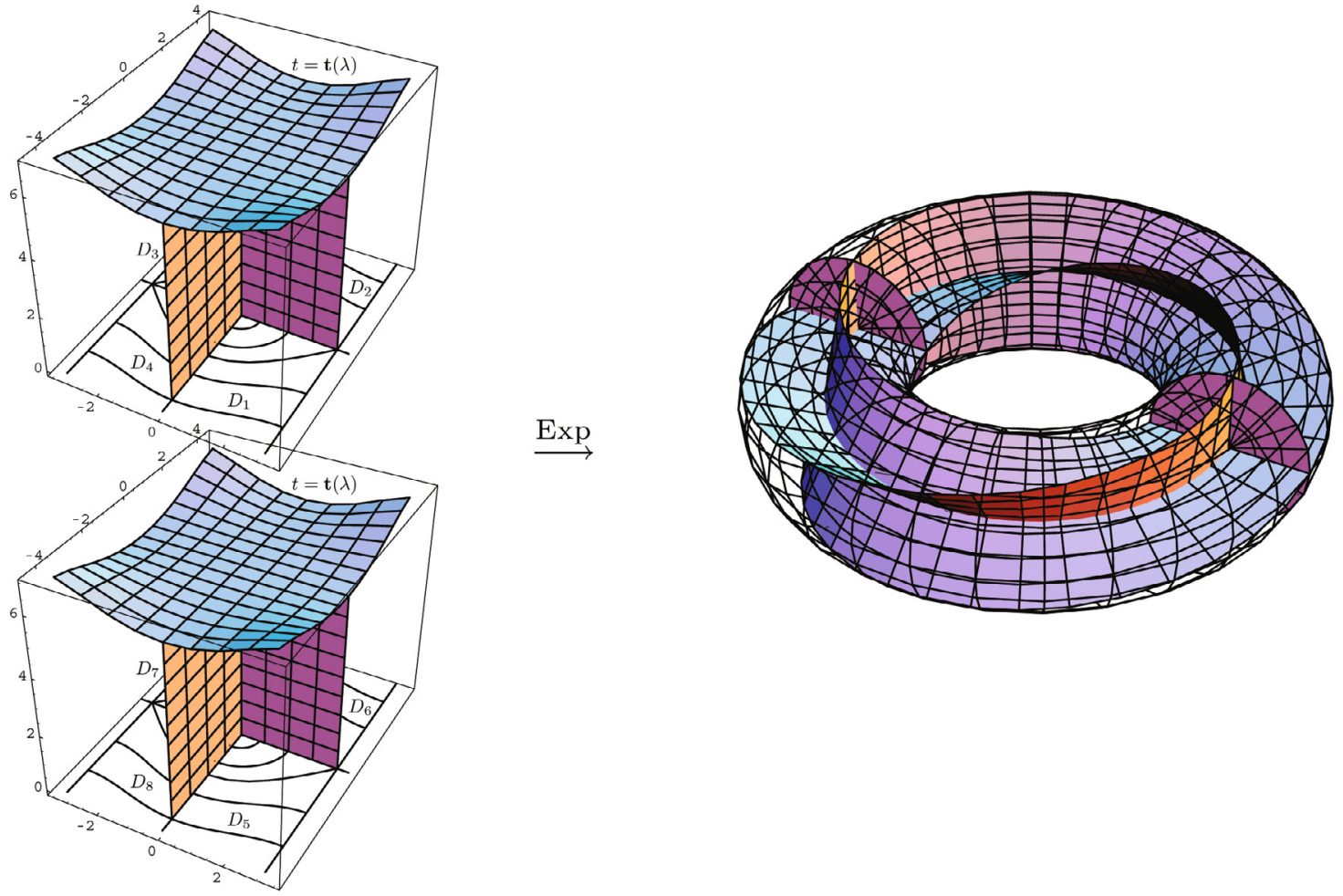

Figure 10. Global structure of exponential mapping.

Then $R_{1}, R_{2}$ become single-valued functions, and the last two rows of Table 2 reflect the signs of these singlevalued functions $R_{i}$ on the sets $M_{i}$.

The boundary $M^{\prime}$ of the domain $\widetilde{M}$ decomposes into four mutually orthogonal surfaces: two planes $\{\theta=0\}$, $\{\theta=\pi\}$ and two Moebius strips $\left\{R_{1}=0\right\},\left\{R_{2}=0\right\}$, see the right-hand side of Figure 10, and Figure 7 [9].

\subsection{Diffeomorphic properties of exponential mapping}

Lemma 3.2. The restriction $\left.\operatorname{Exp}\right|_{\widetilde{N}}$ is nondegenerate.

Proof. If $\nu=(\lambda, t) \in \tilde{N}$, then $t<\mathbf{t}(\lambda)$. By Theorem 2.5, $t_{1}^{\operatorname{conj}}(\lambda) \geq \mathbf{t}(\lambda)$, thus $t<t_{1}^{\operatorname{conj}}(\lambda)=\inf \{s>0 \mid$ $\operatorname{Exp}(\lambda, s)$ is degenerate $\}$. Consequently, the exponential mapping is nondegenerate at the point $(\lambda, t)$.

Lemma 3.3. For any $i=1, \ldots, 8$, we have $\operatorname{Exp}\left(D_{i}\right) \subset M_{i}$.

Proof. We prove only the inclusion $\operatorname{Exp}\left(D_{1}\right) \subset M_{1}$ since the rest inclusions are proved similarly.

Let $(\lambda, t) \in D_{1} \cap N_{1}=\left\{(\lambda, t) \in N_{1} \mid \lambda \in C_{1}^{0}, \tau \in(3 K, 4 K), p \in(0, K), k \in(0,1)\right\}$, see Table 1 . Since $\lambda \in C_{1}^{0}$, then $s_{1}=\operatorname{sgn}\left(\gamma_{t} / 2\right)=1$. Moreover, we have $\operatorname{cn} p \operatorname{sn} p \operatorname{dn} \tau>0$, thus $\sin \theta_{t}<0$ by virtue of equation (5.2) [9]. So $\theta_{t} / 2 \in(\pi / 2, \pi)$. Consequently, $\cos \left(\theta_{t} / 2\right)<0, \sin \theta_{t} / 2>0$ on $D_{1} \cap N_{1}$, thus $s_{3}=-1, s_{4}=1$ in equations $(5.3)-$ (5.6) [9]. Then we get $R_{1}>0$ from equation (5.5) [9], and $R_{2}>0$ from equation (5.6) [9] and Lemma 5.2 [9]. We proved that if $\nu \in D_{1} \cap N_{1}$, then $\sin \theta_{t}<0, R_{1}>0, R_{2}>0$, i.e., $\operatorname{Exp}(\nu) \in M_{1}$, see Table 2. That is, $\operatorname{Exp}\left(D_{1} \cap N_{1}\right) \subset M_{1}$.

A similar argument works in the case $(\lambda, t) \in D_{1} \cap N_{2}=\left\{(\lambda, t) \in N_{2} \mid \lambda \in C_{2}^{+}, \tau \in(-K, 0), p \in\left(0, p_{1}^{1}\right)\right.$, $k \in(0,1)\}$. We have $s_{2}=\operatorname{sgn} c_{t}=1 ; \sin \theta_{t}<0$ by equation (5.7) [9]; $R_{1}>0$ and $R_{2}>0$ by equation (5.10) and (5.11) [9]. Thus $\operatorname{Exp}\left(D_{1} \cap N_{2}\right) \subset M_{1}$. 
It follows from the definition of $N_{3}$ and $N_{2}$ (see Tab. 1) that $D_{1} \cap N_{3} \subset \operatorname{cl}\left(D_{2} \cap N_{3}\right)$, thus $\operatorname{Exp}\left(D_{1} \cap N_{3}\right) \subset$ $\operatorname{cl}\left(M_{1}\right)$. So

$$
\operatorname{Exp}\left(D_{1}\right) \subset \operatorname{cl}\left(M_{1}\right)
$$

Let $\nu=(\lambda, t) \in D_{1} \cap N_{3}$, then $q_{t}=\operatorname{Exp}(\nu) \in \operatorname{cl}\left(M_{1}\right)$. In order to prove that $q_{t} \in M_{1}$, assume by contradiction that $q_{t} \in \partial M_{1}$. By Lemma 3.2, the exponential mapping is a local diffeomorphism near $\nu$. Thus there exist a neighborhood $U \subset D_{1}$ of the point $\nu$ and a neighborhood $V \subset M$ of the point $q_{t}$ such that Exp : $U \rightarrow V$ is a diffeomorphism. Since $q_{t} \in \partial M_{1}$, there exists a point $\widetilde{q} \in V \backslash \operatorname{cl}\left(M_{1}\right)$. Then $\widetilde{\nu}=\operatorname{Exp}^{-1}(\widetilde{q}) \in U \subset D_{1}$, but $\widetilde{q}=\operatorname{Exp}(\widetilde{\nu}) \notin \operatorname{cl}\left(M_{1}\right)$, which contradicts to (3.3). Thus $q_{t} \in M_{1}$. So $\operatorname{Exp}\left(D_{1} \cap N_{3}\right) \subset M_{1}$, and the required inclusion $\operatorname{Exp}\left(D_{1}\right) \subset M_{1}$ follows.

Lemma 3.4. For any $i=1, \ldots, 8$, the mapping $\operatorname{Exp}: D_{i} \rightarrow M_{i}$ is proper.

Proof. Similarly to Lemma 3.1, we can consider only the case $i=2$. Let $K \subset M_{2}$ be a compact, we show that $S=\operatorname{Exp}^{-1}(K) \subset D_{2}$ is a compact as well, i.e., $S$ is bounded and closed.

There exists $\varepsilon>0$ such that

$$
|\sin \theta| \geq \varepsilon, \quad \varepsilon \leq\left|R_{1}\right|,\left|R_{2}\right| \leq 1 / \varepsilon \quad \text { for all } q \in K .
$$

(1) We show that $S$ is bounded. By contradiction, let $\nu_{n}=\left(k_{n}, \varphi_{n}, t_{n}\right) \rightarrow \infty$ for some sequence $\left\{\nu_{n}\right\} \subset S$. Then there exists a sequence $\left\{\nu_{n}\right\} \subset S \cap N_{i}$ for some $i=1,2,3$ with $\nu_{n} \rightarrow \infty$.

Let $S \cap N_{1} \ni \nu_{n}=\left(k_{n}, \varphi_{n}, t_{n}\right) \rightarrow \infty$. Then $t_{n}=2 p_{n} \in\left(0, K\left(k_{n}\right)\right), \tau_{n}=\left(\varphi_{n}+t_{n}\right) / 2 \in\left(0, K\left(k_{n}\right)\right)$. If $k_{n}$ is separated from 1 , then $p_{n}, \tau_{n}$ are bounded, thus $t_{n}, \varphi_{n}$ are bounded, a contradiction. Thus $k_{n} \rightarrow 1$ for a subsequence (we will assume that this holds for the initial sequence).

If $\left(\gamma_{n}, c_{n}\right) \rightarrow( \pm \pi, 0)$, then $\left(\theta_{t}, y_{t}\right) \rightarrow 0$, thus $R_{1} \rightarrow 0$, a contradiction. Thus the sequence $\left(\gamma_{n}, c_{n}\right)$ is separated from the point $( \pm \pi, 0)$.

Then there exists a sequence such that $k_{n} \rightarrow 1$ and $\varphi_{n} \rightarrow \varphi \in(-\infty,+\infty)$, thus $t_{n} \rightarrow+\infty, p_{n} \rightarrow+\infty$, $\tau_{n} \rightarrow+\infty$. Then $\left(p_{n}-\mathrm{E}\left(p_{n}\right)\right) /\left(k_{n} \sqrt{\Delta}\right) \rightarrow \infty, f_{2}\left(p_{n}, k_{n}\right) /\left(k_{n} \sqrt{\Delta}\right) \rightarrow \infty$.

If $\operatorname{cn}\left(\tau_{n}\right)$ is separated from zero, then $R_{1} \rightarrow \infty$ (see equation (5.5) [9]). And if $\operatorname{cn}\left(\tau_{n}\right)$ is not separated from zero, then there exists a sequence such that $\operatorname{cn}\left(\tau_{n}\right) \rightarrow 0$, thus $\operatorname{sn}\left(\tau_{n}\right)$ is separated from zero, then $R_{2} \rightarrow \infty$ (see equation (5.6) [9]).

So the hypothesis $S \cap C_{1} \ni \nu_{n}=\left(k_{n}, \varphi_{n}, t_{n}\right) \rightarrow \infty$ leads to a contradiction.

Similarly the hypotheses $C \cap C_{i} \ni \nu_{n} \rightarrow \infty, i=2,3$, lead to a contradiction.

Thus the set $S=\operatorname{Exp}^{-1}(K)$ is bounded.

(2) We show that $S$ is closed. Let $\left\{\nu_{n}\right\} \subset S$, we have to prove that there exists a subsequence $\nu_{n_{k}}$ converging in $D_{2}$. By contradiction, let $\nu_{n} \rightarrow \infty$ or $\nu_{n} \rightarrow \nu \in \partial D_{2}$.

Consider the case $\nu_{n}=\left(\tau_{n}, p_{n}, k_{n}\right) \in S \cap N_{1}$.

If $k_{n} \rightarrow 0$, then $(x, y) \rightarrow 0$, thus $R_{1}, R_{2} \rightarrow 0$, a contradiction.

Let $k_{n} \rightarrow 1$. If $\left(\gamma_{n}, c_{n}\right) \rightarrow( \pm \pi, 0)$, then $(\theta, y) \rightarrow(0,0)$, thus $R_{1} \rightarrow 0$, a contradiction.

If $\left(\gamma_{n}, c_{n}\right) \rightarrow(\gamma, c) \neq( \pm \pi, 0)$, then $\nu \in N_{3}$, a contradiction.

Thus $k_{n} \rightarrow k \in(0,1)$. Then $\tau_{n} \rightarrow \tau \in[3 K(k), 4 K(k)]$. If $\tau=3 K$, then $R_{1} \rightarrow 0$, and if $\tau=4 K$, then $R_{2} \rightarrow 0$, a contradiction. Thus $\tau_{n} \rightarrow \tau \in(3 K(k), 4 K(k))$.

Further, $p_{n} \rightarrow p \in[0, K(k)]$. If $p=0$, then $t=0$ and $R_{1}, R_{2} \rightarrow 0$. If $p=K$, then $R_{1} \rightarrow 0$. Thus $p_{n} \rightarrow p \in(0, K)$.

So $\left(\tau_{n}, p_{n}, k_{n}\right) \rightarrow(\tau, p, k) \in N_{1}$, a contradiction.

We proved that any sequence $\nu_{n} \in S \cap N_{1}$ contains a subsequence converging in $D_{2}$. Similarly one proves the same for a sequence $\nu_{n} \in S \cap N_{i}, i=2,3$.

Thus any sequence $\nu_{n} \in S$ contains a subsequence converging in $D_{2}$, thus converging in $S$. So the set $S=\operatorname{Exp}^{-1}(K)$ is closed. 
Theorem 3.1. For any $i=1, \ldots, 8$, we have $\operatorname{Exp}\left(D_{i}\right) \subset M_{i}$, and the mapping $\operatorname{Exp}: D_{i} \rightarrow M_{i}$ is a diffeomorphism.

Proof. The inclusion $\operatorname{Exp}\left(D_{i}\right) \subset M_{i}$ was proved in Lemma 3.3. The mapping $\operatorname{Exp}: D_{i} \rightarrow M_{i}$ is smooth, nondegenerate (Lem. 3.2), and proper (Lem. 3.4), thus it is a covering. Since $M_{i}$ is simply connected, the mapping $\operatorname{Exp}: D_{i} \rightarrow M_{i}$ is a diffeomorphism.

Lemma 3.5. $\operatorname{Exp}\left(N_{4}\right)=\left\{q \in M \mid R_{1}=R_{2}=0\right\}=\{q \in M \mid x=y=0\}, \operatorname{Exp}\left(N_{5}\right)=\left\{q \in M \mid R_{1}=0\right.$ $\left.R_{2} \neq 0, \theta=0\right\}=\{q \in M \mid x \neq 0, y=0, \theta=0\}$.

Proof. Follows immediately from the corresponding formulas for extremal trajectories of Section 3.3 [9].

Lemma 3.6. $\operatorname{Exp}\left(N^{\prime}\right) \subset M^{\prime}$.

Proof. Follows from formulas (5.2)-(5.11) [9].

Theorem 3.1 implies the following statement.

Corollary 3.1. The mapping $\operatorname{Exp}: \widetilde{N} \rightarrow \widetilde{M}$ is a diffeomorphism.

In view of Lemma 3.6, for any $q \in \widetilde{M}$ there exists a unique $\nu=(\lambda, t)=\operatorname{Exp}^{-1}(q) \in \widetilde{N}, \lambda=\lambda(q), t=t(q)$.

The diffeomorphism Exp : $\widetilde{N}=\cup_{i=1}^{8} D_{i} \rightarrow \widetilde{M}=\cup_{i=1}^{8} M_{i}$ is schematically shown at Figure 10 . At this figure in the left, each set $N_{i}, i=1, \ldots, 5$, projects down to the corresponding subset $C_{i}$ in the phase cylinder of the pendulum, see (1.8)-(1.12) and Figure 2 [9]. Each domain $D_{i}, i=1, \ldots, 8$, is mapped to one of the domains $M_{i}, i=1, \ldots, 8$, cut out in the solid torus by the $\operatorname{discs}\{\theta=0\},\{\theta=\pi\}$, and the Moebius strips $\left\{R_{1}=0\right\},\left\{R_{2}=0\right\}$. Boundaries of the domains $D_{i}$ are mapped to these discs and Moebius strips in a more complicated way to be described in detail in the forthcoming work [19].

\subsection{Cut time}

Theorem 3.2. For any $q_{1} \in \widetilde{M}$, let $\left(\lambda_{1}, t_{1}\right)=\operatorname{Exp}^{-1}\left(q_{1}\right) \in \widetilde{N}$. Then the extremal trajectory $q(s)=\operatorname{Exp}\left(\lambda_{1}, s\right)$ is optimal with $q\left(t_{1}\right)=q_{1}$.

Thus optimal synthesis on the domain $\widetilde{M}$ is given by

$$
u_{i}(q)=h_{i}(\lambda), \quad i=1,2, \quad(\lambda, t)=\operatorname{Exp}^{-1}(q) \in \widetilde{N}, \quad q \in \widetilde{M}
$$

Proof. Let $q_{1} \in \widetilde{M}$. There exists $\nu_{1}=\left(\lambda_{1}, t_{1}\right) \in \widehat{N}=\widetilde{N} \sqcup N^{\prime}$ such that the trajectory $q(s)=\operatorname{Exp}\left(\lambda_{1}, s\right)$ is optimal and $q\left(t_{1}\right)=\operatorname{Exp}\left(\nu_{1}\right)=q_{1}$. By Lemmas 3.5 and 3.6, we have $\nu_{1} \in \tilde{N}$. By Corollary 3.1, there exists a unique $\nu_{1} \in \widetilde{N}$ such that $\operatorname{Exp}\left(\nu_{1}\right)=q_{1}$. So $q(s)=\operatorname{Exp}\left(\lambda_{1}, s\right)$ is a unique optimal trajectory coming to $q_{1}$.

In work [9] we proved the inequality $t_{\text {cut }}(\lambda) \leq \mathbf{t}(\lambda)$. Now we prove the corresponding equality.

Theorem 3.3. For any $\lambda \in C$ we have $t_{\text {cut }}(\lambda)=\mathbf{t}(\lambda)$.

Proof. We proved the inequality $t_{\text {cut }}(\lambda) \leq \mathbf{t}(\lambda)$ in Theorem $5.4[9]$.

(1) Consider first the generic case $\lambda_{1} \in \cup_{i=1}^{3} C_{i}$. There exists $t_{1} \in\left(0, \mathbf{t}\left(\lambda_{1}\right)\right)$ and arbitrarily close to $\mathbf{t}\left(\lambda_{1}\right)$ such that $\operatorname{sn} \tau_{1} \operatorname{cn} \tau_{1} \neq 0$. Then $\nu_{1}=\left(\lambda_{1}, t_{1}\right) \in \widetilde{N}$, thus $q_{1}=\operatorname{Exp}\left(\nu_{1}\right) \in \widetilde{M}$. By Theorem 3.2, the trajectory $q(s)=\operatorname{Exp}\left(\lambda_{1}, s\right), s \in\left[0, t_{1}\right]$, is optimal, thus $t_{1} \leq t_{\text {cut }}\left(\lambda_{1}\right)$.

So there exists $t_{1} \in\left(0, \mathbf{t}\left(\lambda_{1}\right)\right)$ arbitrarily close to $\mathbf{t}\left(\lambda_{1}\right)$ such that $t_{1} \leq t_{\text {cut }}\left(\lambda_{1}\right)$. Consequently, $\mathbf{t}\left(\lambda_{1}\right) \leq t_{\text {cut }}\left(\lambda_{1}\right)$.

We proved that $t_{\text {cut }}\left(\lambda_{1}\right)=\mathbf{t}\left(\lambda_{1}\right)$ for any $\lambda_{1} \in \cup_{i=1}^{3} C_{i}$.

(2) If $\lambda \in C_{4}$, then the extremal trajectory $(x, y, \theta)=(0,0, \pm t)$ is a Riemannian geodesic for the restriction of the sub-Riemannian problem on $\operatorname{SE}(2)$ to the circle $\left\{(0,0, \theta) \mid \theta \in S^{1}\right\}$. It is optimal up to the antipodal point, thus $t_{\text {cut }}(\lambda)=\pi=\mathbf{t}(\lambda)$.

(3) In the case $\lambda \in C_{5}$ the extremal trajectory is a line $(x, y, \theta)=( \pm t, 0,0)$, thus it is optimal forever: $t_{\text {cut }}(\lambda)=+\infty=\mathbf{t}(\lambda)$. 


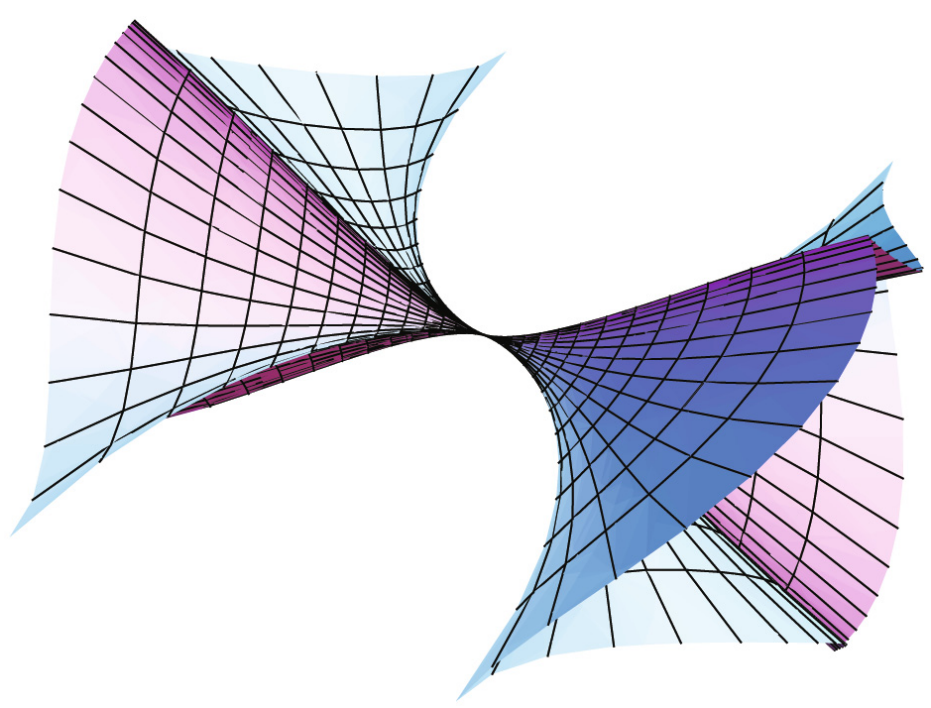

FiguRE 11. Sub-Riemannian caustic.

\section{Plots of sub-Riemannian CAustic And SPheres}

Here we collect 3-dimensional plots of some essential objects in the sub-Riemannian problem on $\mathrm{SE}(2)$.

Figure 11 shows the sub-Riemannian caustic

$$
\left\{\operatorname{Exp}(\lambda, t) \mid \lambda \in C, t=t_{1}^{\operatorname{conj}}(\lambda)\right\}
$$

in the rectifying coordinates $\left(R_{1}, R_{2}, \theta\right)$.

Figures 12-20 present sub-Riemannian spheres

$$
S_{R}=\left\{q \in M \mid d\left(q_{0}, q\right)=R\right\}=\left\{\operatorname{Exp}(\lambda, R) \mid \lambda \in C, t_{\text {cut }}(\lambda) \geq R\right\}
$$

of different radii $R$, where

$$
d\left(q_{0}, q_{1}\right)=\inf \left\{l(q(\cdot)) \mid q(\cdot) \text { trajectory of }(1.1), q(0)=q_{0}, q\left(t_{1}\right)=q_{1}\right\}
$$

is the sub-Riemannian distance - the cost function in the sub-Riemannian problem (1.1)-(1.5).

In this problem sub-Riemannian spheres can be of three different topological classes. If $R \in(0, \pi)$, then $S_{R}$ is homeomorphic to the standard 2-dimensional Euclidean sphere $S^{2}$, see Figure 14. For $R=\pi$ the sphere $S_{R}$ is homeomorphic to the sphere $S^{2}$ with its north pole $N$ and south pole $S$ identified: $S_{\pi} \cong S^{2} /\{N=S\}$, see Figure 17. And if $R>\pi$, then $S_{R}$ is homeomorphic to the 2-dimensional torus, see Figure 20.

Figure 12 shows the sphere $S_{\pi / 2}$ in rectifying coordinates $\left(R_{1}, R_{2}, \theta\right)$. Figure 13 represents the same sphere with a cut-out opening the singularities of the sphere: the sphere intersects itself at the local components of the cut locus Cut loc described in [19]. Figure 14 shows embedding of the same sphere to the solid torus. Sub-Riemannian spheres of small radii resemble the well-known apple-shaped sub-Riemannian sphere in the Heisenberg group [21]. Although, there is a major difference: the sphere in the Heisenberg group has a oneparameter family of symmetries (rotations), but the sphere in $\mathrm{SE}(2)$ has only a discrete group of symmetries $G=\left\{\mathrm{Id}, \varepsilon^{1}, \ldots, \varepsilon^{7}\right\}$ (reflections). 


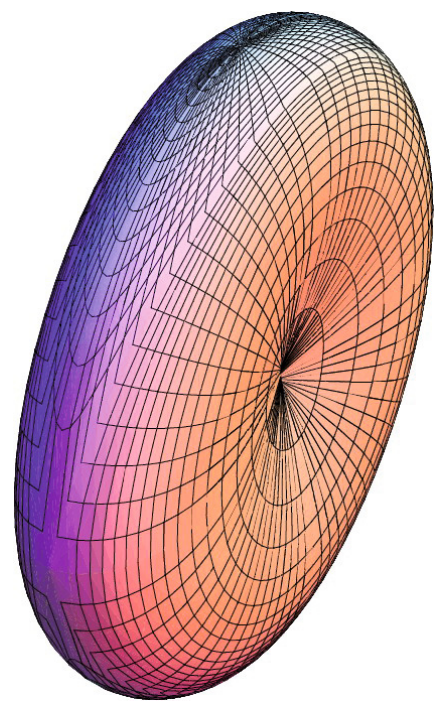

Figure 12. Sub-Riemannian sphere $S_{\pi / 2}$.

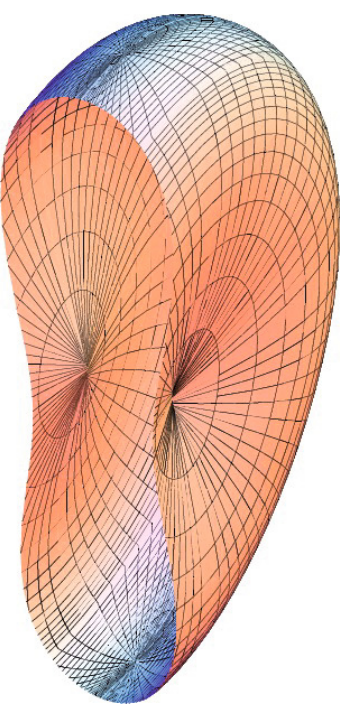

Figure 13. Sub-Riemannian sphere $S_{\pi / 2}$ with cut-out.

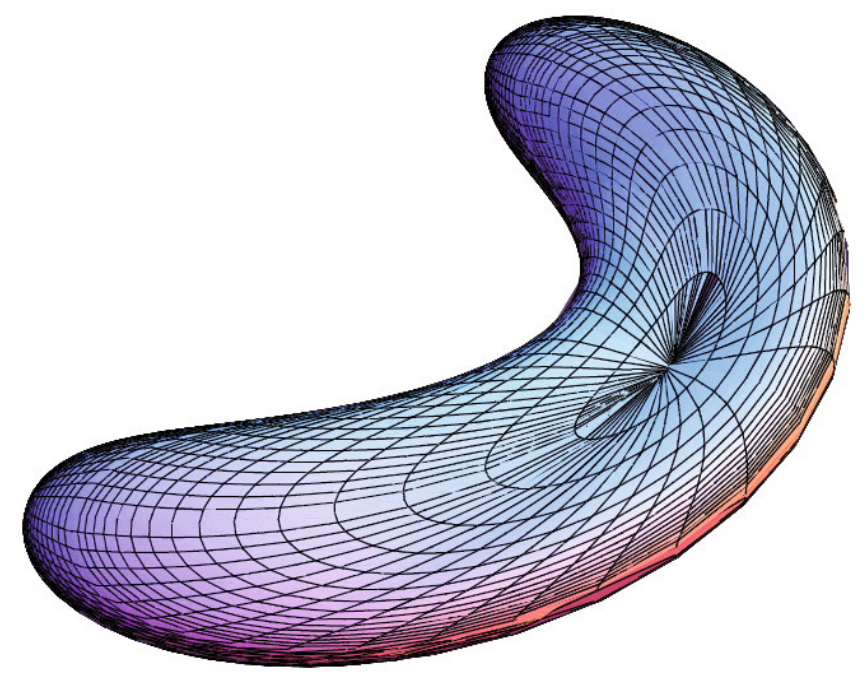

FiguRE 14. Sub-Riemannian sphere $S_{\pi / 2}$, global view.

Figures 15-17 represent similarly the sub-Riemannian sphere of the critical radius $\pi$. In addition to selfintersections at the local component $\mathrm{Cut}_{\text {loc }}$, the sphere $S_{\pi}$ has one self-intersection point at the global component Cut glob.

Figures 18-20 show similar images of the sphere of radius $3 \pi / 2$. For $R>\pi$ the sphere $S_{R}$ has two topological segments of self-intersection points at $\mathrm{Cut}_{\mathrm{loc}}^{ \pm}$respectively, and a topological circle of self-intersection points at Cut glob.

Figures 21 and 22 show intersections of the spheres $S_{\pi / 2}, S_{\pi}, S_{3 \pi / 2}$ with the half-spaces $\theta<0$ and $R_{2}>0$ respectively. 


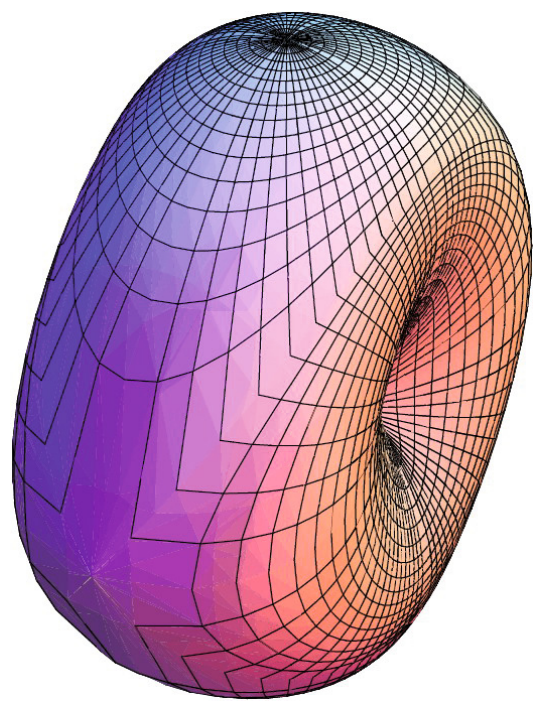

FiguRE 15. Sub-Riemannian sphere $S_{\pi}$.

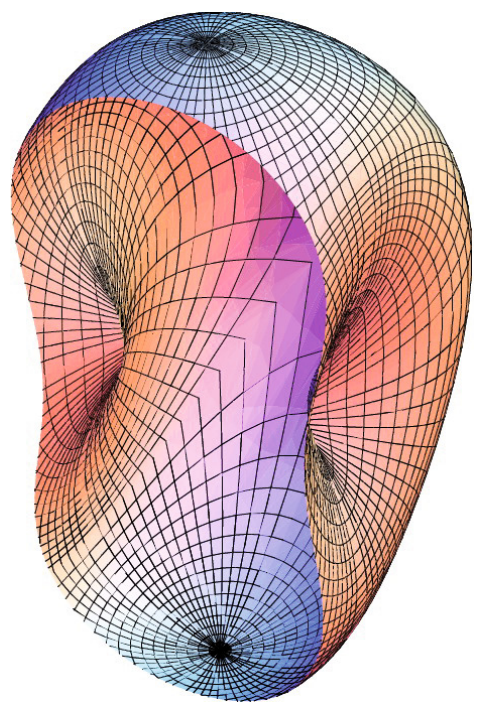

Figure 16. Sub-Riemannian sphere $S_{\pi}$ with cut-out.

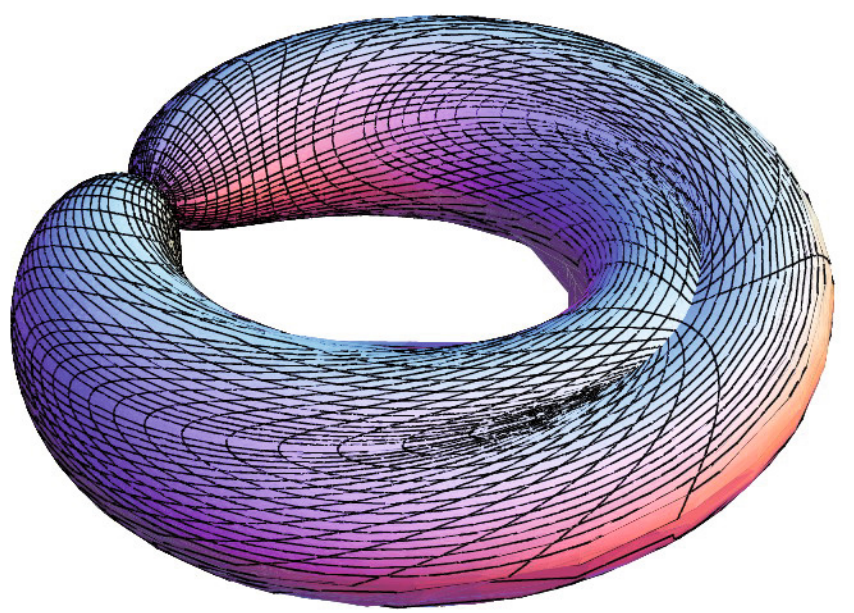

Figure 17. Sub-Riemannian sphere $S_{\pi}$, global view.

Figure 23 shows self-intersections of the wavefront

$$
W_{R}=\{\operatorname{Exp}(\lambda, R) \mid \lambda \in C\}
$$

for $R=\pi$.

\section{Conclusion}

The solution to the sub-Riemannian problem on $\mathrm{SE}(2)$ obtained in the previous paper [9], this one, and the subsequent paper [19] is based on a detailed study of the action of the discrete group of symmetries of the exponential mapping. This techniques was already partially developed in the study of related optimal 


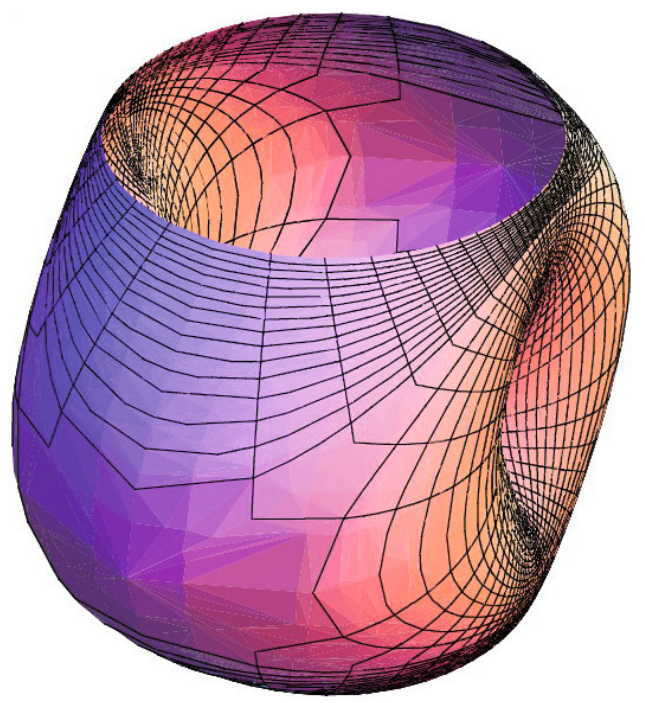

FiguRE 18. Sub-Riemannian sphere $S_{3 \pi / 2}$.

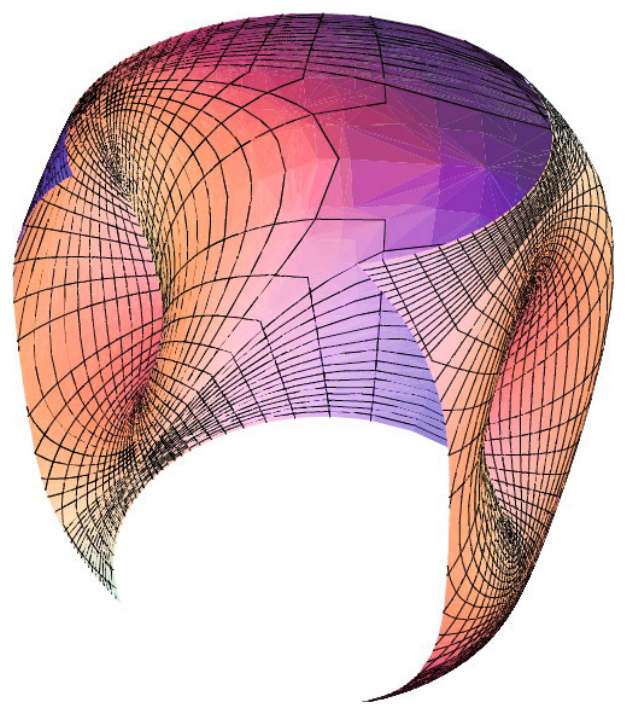

FiguRE 19. Sub-Riemannian sphere $S_{3 \pi / 2}$ with cut-out.

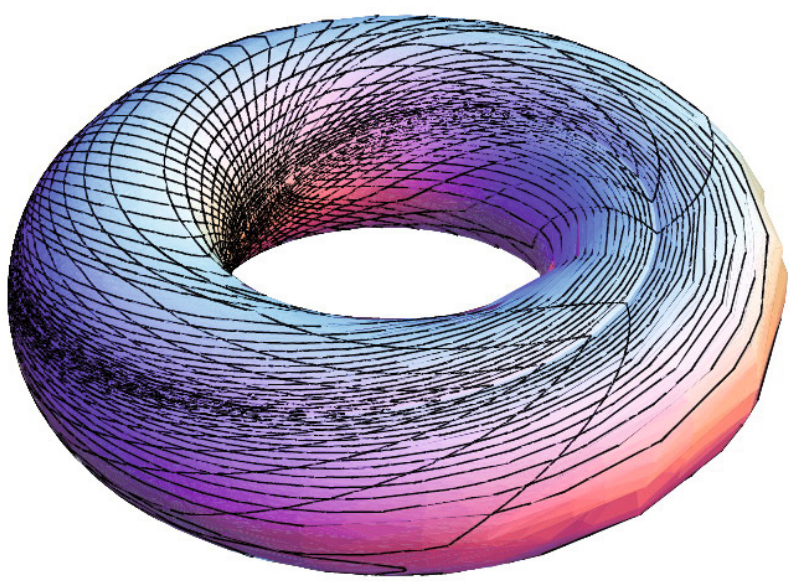

Figure 20. Sub-Riemannian sphere $S_{3 \pi / 2}$, global view.

control problems (nilpotent sub-Riemannian problem with the growth vector $(2,3,5)$ [13-16] and Euler's elastic problem $[17,18])$. The sub-Riemannian problem on $\mathrm{SE}(2)$ is the first problem in this series, where a complete solution was obtained (local and global optimality, cut time and cut locus, optimal synthesis). We believe that our approach based on the study of symmetries will provide such complete results in other symmetric invariant problems, such as the nilpotent sub-Riemannian problem with the growth vector $(2,3,5)$, the ball-plate problem [7], and others. 


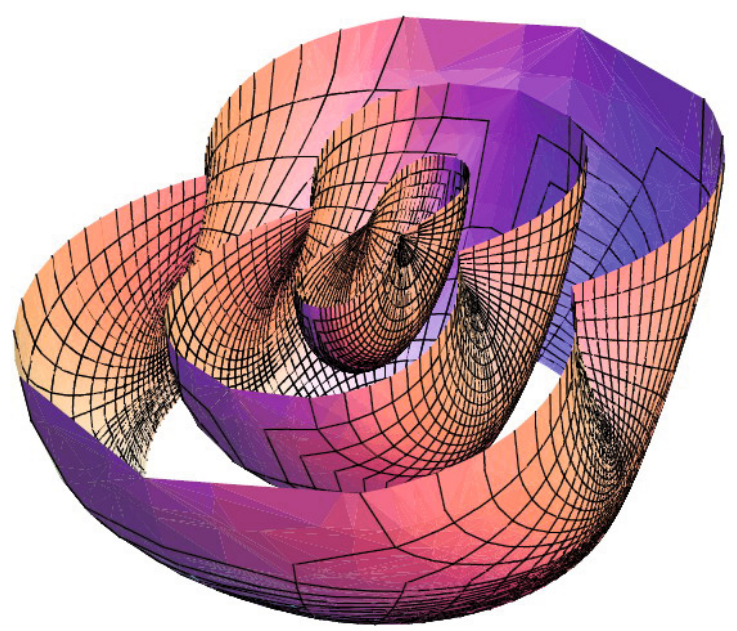

Figure 21. Sub-Riemannian hemi-spheres $S_{\pi / 2}, S_{\pi}, S_{3 \pi / 2}$ for $\theta<0$.

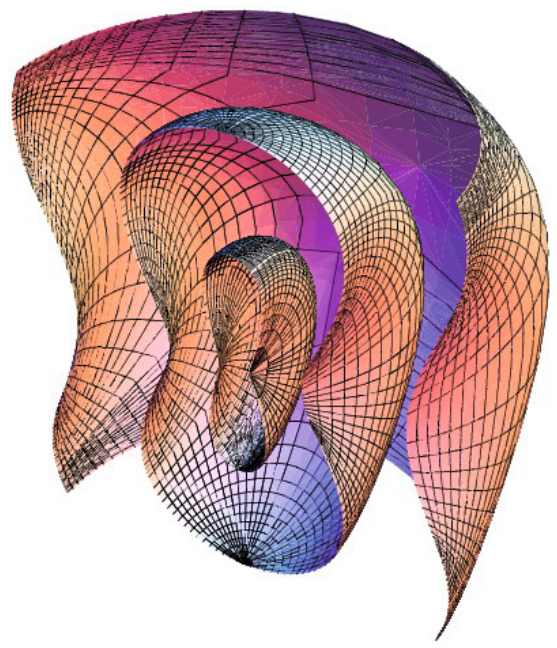

Figure 22. Sub-Riemannian hemi-spheres $S_{\pi / 2}, S_{\pi}, S_{3 \pi / 2}$ for $R_{2}>0$.

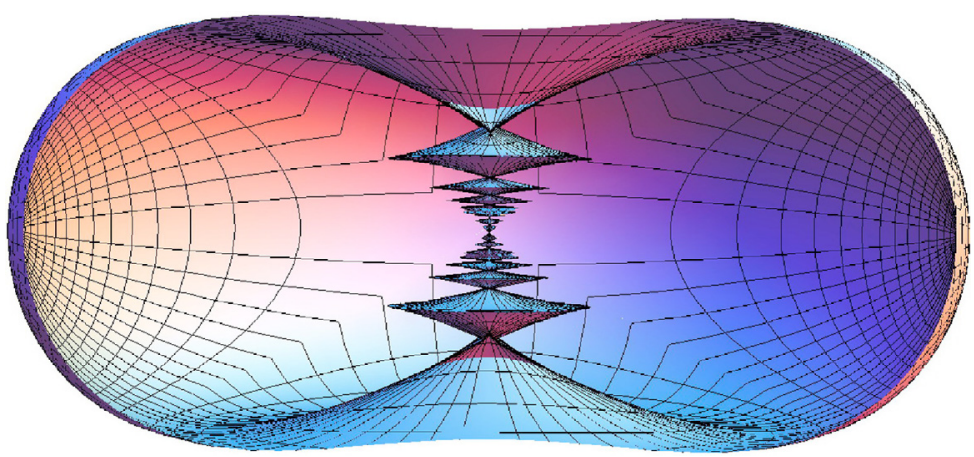

Figure 23. Wavefront $W_{\pi}$ with cut-out. 
Acknowledgements. The author is grateful to Prof. Andrei Agrachev for bringing the sub-Riemannian problem on SE(2) to authors' attention. The author wishes to thank Prof. Arrigo Cellina for hospitality and excellent conditions for work at the final version of this paper in University Milano Bicocca. The author thanks an anonymous reviewer whose valuable comments improved essentially presentation of this work.

\section{REFERENCES}

[1] A.A. Agrachev, Exponential mappings for contact sub-Riemannian structures. J. Dyn. Control Syst. 2 (1996) 321-358.

[2] A.A. Agrachev, Geometry of optimal control problems and Hamiltonian systems, in Nonlinear and Optimal Control Theory, Lect. Notes Math. CIME 1932, Springer Verlag (2008) 1-59.

[3] A.A. Agrachev and Y.L. Sachkov, Control Theory from the Geometric Viewpoint. Springer-Verlag, Berlin (2004).

[4] A.A. Agrachev, U. Boscain, J.P. Gauthier and F. Rossi, The intrinsic hypoelliptic Laplacian and its heat kernel on unimodular Lie groups. J. Funct. Anal. 256 (2009) 2621-2655.

[5] G. Citti and A. Sarti, A cortical based model of perceptual completion in the roto-translation space. J. Math. Imaging Vis. 24 (2006) 307-326.

[6] C. El-Alaoui, J.P. Gauthier and I. Kupka, Small sub-Riemannian balls on $\mathbb{R}^{3}$. J. Dyn. Control Syst. 2 (1996) $359-421$.

[7] V. Jurdjevic, Geometric Control Theory. Cambridge University Press (1997).

[8] J.P. Laumond, Nonholonomic motion planning for mobile robots, Lecture Notes in Control and Information Sciences 229. Springer (1998).

[9] I. Moiseev and Y.L. Sachkov, Maxwell strata in sub-Riemannian problem on the group of motions of a plane. ESAIM: COCV (2009), doi:10.1051/cocv/2009004.

[10] J. Petitot, The neurogeometry of pinwheels as a sub-Riemannian contact structure. J. Physiology - Paris 97 (2003) 265-309.

[11] J. Petitot, Neurogéometrie de la vision - Modèles mathématiques et physiques des architectures fonctionnelles. Éditions de l'École polytechnique, France (2008).

[12] L.S. Pontryagin, V.G. Boltyanskii, R.V. Gamkrelidze and E.F. Mishchenko, The mathematical theory of optimal processes. Wiley Interscience (1962).

[13] Y.L. Sachkov, Exponential mapping in generalized Dido's problem. Mat. Sbornik 194 (2003) 63-90 (in Russian). English translation in Sbornik: Mathematics 194 (2003).

[14] Y.L. Sachkov, Discrete symmetries in the generalized Dido problem. Matem. Sbornik 197 (2006) 95-116 (in Russian). English translation in Sbornik: Mathematics, 197 (2006) 235-257.

[15] Y.L. Sachkov, The Maxwell set in the generalized Dido problem. Matem. Sbornik 197 (2006) 123-150 (in Russian). English translation in Sbornik: Mathematics 197 (2006) 595-621.

[16] Y.L. Sachkov, Complete description of the Maxwell strata in the generalized Dido problem. Matem. Sbornik 197 (2006) 111-160 (in Russian). English translation in: Sbornik: Mathematics 197 (2006) 901-950.

[17] Y.L. Sachkov, Maxwell strata in Euler's elastic problem. J. Dyn. Control Syst. 14 (2008) 169-234.

[18] Y.L. Sachkov, Conjugate points in Euler's elastic problem. J. Dyn. Control Syst. 14 (2008) 409-439.

[19] Y.L. Sachkov, Cut locus and optimal synthesis in sub-Riemannian problem on the group of motions of a plane. ESAIM: COCV (submitted).

[20] A.V. Sarychev, The index of second variation of a control system. Matem. Sbornik 113 (1980) 464-486 (in Russian). English translation in Math. USSR Sbornik 41 (1982) 383-401.

[21] A.M. Vershik and V.Y. Gershkovich, Nonholonomic Dynamical Systems - Geometry of distributions and variational problems (in Russian), in Itogi Nauki i Tekhniki: Sovremennye Problemy Matematiki, Fundamental'nyje Napravleniya 16, VINITI, Moscow (1987) 5-85. English translation in Encyclopedia of Math. Sci. 16, Dynamical Systems 7, Springer Verlag.

[22] E.T. Whittaker and G.N. Watson, A Course of Modern Analysis. An introduction to the general theory of infinite processes and of analytic functions; with an account of principal transcendental functions. Cambridge University Press, Cambridge (1996).

[23] S. Wolfram, Mathematica: a system for doing mathematics by computer. Addison-Wesley, Reading, USA (1991). 